\title{
Flexible Spatial Configuration of Local Image Features
}

\author{
Gustavo Carneiro and Allan D. Jepson, Member, IEEE Computer Society
}

\begin{abstract}
Local image features have been designed to be informative and repeatable under rigid transformations and illumination deformations. Even though current state-of-the-art local image features present a high degree of repeatability, their local appearance alone usually does not bring enough discriminative power to support a reliable matching, resulting in a relatively high number of mismatches in the correspondence set formed during the data association procedure. As a result, geometric filters, commonly based on global spatial configuration, have been used to reduce this number of mismatches. However, this approach presents a trade-off between the effectiveness to reject mismatches and the robustness to nonrigid deformations. In this paper, we propose two geometric filters, based on a semilocal spatial configuration of local features, that are designed to be robust to nonrigid deformations and to rigid transformations, without compromising its efficacy to reject mismatches. We compare our methods to the Hough transform, which is an efficient and effective mismatch rejection step based on the global spatial configuration of features. In these comparisons, our methods are shown to be more effective in the task of rejecting mismatches for rigid transformations and nonrigid deformations at comparable time complexity figures. Finally, we demonstrate how we can integrate these methods in a probabilistic recognition system such that the final verification step uses not only the similarity between features but also their semilocal configuration.
\end{abstract}

Index Terms-Local image feature, feature clustering, visual object recognition, wide baseline matching, long-range matching.

\section{INTRODUCTION}

$\mathrm{T}$ HE field of computer vision has experienced an increasing interest in the use of local image features for the tasks of object recognition [25], image matching [33], object discovery and recognition [38], and so forth. When compared to image representations based on a large spatial support [29], local feature representations (based on a small spatial support) trade a poorer distinctiveness for a better robustness to brightness deformations and rigid transformations. Therefore, the search for similar features between the local features extracted from a test image and the features in the model database typically returns a correspondence set with a high percentage of mismatches. The rejection of mismatches from this correspondence set is therefore one of the central issues in local-feature-based methods for recognition.

The rejection of mismatches is typically based on the spatial configuration of the model features. The global spatial configuration (for example, [16], [23], [25], [32], [39], and [43]) assumes that all model features suffered a rigid transformation. Usually, the more strict this assumption of global transform is, the more effective the method is to reject mismatches. As this assumption is relaxed, the method becomes more robust to nonrigid deformations but allows more mismatches in the correspondence set. A more flexible scheme was introduced by Berg et al. [5], which alleviates this

- G. Carneiro is with Siemens Corporate Research, Integrated Data Systems Department, 755 College Road East, Princeton, NJ 08540.

E-mail: gustavo.carneiro@siemens.com.

- A.D. Jepson is with the Department of Computer Science, D.L. Pratt Building, Rm 283C, 6 King's College Rd., University of Toronto, Toronto, Ontario M5S3H5 Canada. E-mail: jepson@cs.utoronto.ca.

Manuscript received 11 Mar. 2006; revised 14 Nov. 2006; accepted 5 Feb. 2007; published online 6 Mar. 2007.

Recommended for acceptance by L. Van Gool.

For information on obtaining reprints of this article, please send e-mail to: tpami@computer.org, and reference IEEECS Log Number TPAMI-0197-0306. Digital Object Identifier no. 10.1109/TPAMI.2007.1126.

0162-8828/07/\$25.00 (C) 2007 IEEE problem by allowing some flexibility to the initial rigid model through the use of thin plate splines, but the trade-off mentioned above is still present. A method specifically designed to be robust to nonrigid deformations was presented by Ferrari et al. [17], where the authors propose an algorithm consisting of several steps of expansion and contraction of the correspondence set that slowly rejects mismatches and increases the number of correct correspondences. The main issue with the latter method is the high computational complexity of the whole algorithm. A method for real-time tracking of nonrigid surfaces is proposed by Pilet et al. [30], where the method is based on deformable 2D meshes and the use of robust estimators. This system produces impressive nonrigid matching results at relatively high frame rates (10 fps), but the main problem with the method is the difficulty in matching highly deformable objects because of issues involved in the minimization of the surface energy term. Here, we propose two efficient methods to reject mismatches that are designed to be robust to nonrigid deformations, but for which the rejection of mismatches from the correspondence set is less affected. Specifically, the following methods are considered: 1) the introduction of an intermediate grouping step using pairwise geometric relations [9] and 2) the improvement of the distinctiveness of the local feature using semilocal geometric information [10]. We also propose a novel probabilistic verification method based on feature similarity and semilocal geometric relations. This verification method can be combined with either mismatch rejection methods 1 or 2 above to increase the proportion of correct matches in the correspondence set and also to verify the correctness of the semilocal geometric configuration of the features.

We present a comparison between both mismatch rejection methods and Hough clustering, which is a common method to reject mismatches based on the global spatial configuration. The results show that both methods lead to correspondence sets with a higher proportion of 
correct matches than Hough clustering for both rigid transformations and nonrigid deformations. We also show that our methods present a comparable time complexity when compared to Hough clustering for typical image matching tasks. The probabilistic verification method that uses semilocal geometric relations is shown to increase the ratio of correct matches in the final correspondence set, without increasing the total time complexity for rejecting mismatches. Finally, we show how these methods can be combined in a recognition system, where we show results on wide baseline stereo and long-range matching problems.

\section{Literature ReView}

Systems that exploit pairwise relations to reject mismatches can be traced back to [3], [7], [24]. In [27], [45], pairwise relations were used to disambiguate matches, but both papers rely on a verification stage that is based on a global transformation, which is not suitable to handle nonrigid deformations. Yu et al. [44] exploit pairwise relations of parts, but compared to our approach, their method can work with 5 to 10 parts only, whereas our method can handle hundreds of parts. The use of graphs is exploited by Dickinson et al. [14], [36], [37], where objects are represented as a hierarchical graph and the matching process takes into account the graph structure, the (semi) local features, and their global spatial arrangements. Huet and Hancock [22] introduce an approach where the features are based only on pairwise geometric relations between lines in a structural representation of objects and impressive recognition results are obtained, showing that pairwise relations alone can represent a powerful indexing feature. Even though the use of pairwise relations is generally associated with mismatch rejection methods, they can also be exploited in the verification stage, as implemented by Agarwal and Roth [1].

The use of semilocal information to enhance the discriminating power of local features has also been exploited in the literature. The most relevant work for our approach was presented by Schmid and Mohr [34], [35], where a fixed number of local features around a given feature are used to determine its semilocal structure. Also, a method similar to ours has been recently proposed by Mortensen et al. [28]. A slightly different approach to eliminate mismatches is proposed by Schaffalitzky and Zisserman [33], where a neighborhood consensus, formed by a fixed number of features, is imposed to reject mismatches. Semilocal constraints are also used by Tuytelaars and Van Gool [42], where an iterative method rejects mismatches based on homographies between matches of semilocal features. Tell and Carlsson [40] introduce a semilocal feature formed by a group of ordered local features, which improves the discriminating power of the feature, but even though an optimal algorithm is used to avoid all possible combinations of neighboring local features, the method is still prohibitively complex. Parts and a union of parts are exploited by Huang et al. [12], where the parts are described as polynomial surfaces. This approach represents both semilocal and global features, since the union of parts can represent the whole object, but articulated objects are not handled properly, given that the relations are assumed to be fixed between the parts. The use of pairwise relations to form a feature vector is also successfully used by Belongie et al. [4], where the authors propose the semilocal feature shape context. The method proposed by Amit and Geman [2] learns groups of a fixed number of local features (thus forming semilocal features) for recognition. Finally, Chum et al. [13] show that the use of three point correspondences (or regions) within a RAndom SAmple Consensus (RANSAC) loop to estimate the F matrix speeds up the estimation of the epipolar geometry and allows for a higher robustness to mismatches.

The novelty of our approaches lies in the use of the semilocal configuration of features for rejecting mismatches and verifying hypotheses, which means that we never rely on the global configuration of local features. Both mismatch rejection methods proposed here build the semilocal configuration by using all of the image features (as opposed to a fixed number of features) in a tunable neighborhood (the size of this neighborhood is a user-defined parameter). Moreover, the feature and semilocal similarity functions are combined in the verification step by using probabilistic measures, thus avoiding the hard task of determining a reasonable similarity function involving these rather distinct similarity functions. Also, our methods are capable of handling correspondence sets containing thousands of pairings efficiently. Finally, similarly to [45], our approach weights the importance of a semilocal geometrical correspondence by its scale-invariant pairwise distance, meaning that nearby features are more likely to preserve such similarities than far away features.

\section{Local Image Features}

A local feature is represented by a geometric characterization of an image region plus a descriptor of the image function (photometry) of this region. More specifically, a local feature vector is described as $\mathbf{f}_{l}=\left[m_{l}, \mathbf{x}_{l}, \theta_{l}, \sigma_{l}, \mathbf{v}_{l}\right]$, where $m_{l}$ is the model identification, $\mathbf{x}_{l}$ is the spatial position of the feature, $\theta_{l}$ represents the dominant orientation at position $\mathbf{x}_{l}, \sigma_{l}$ denotes the feature scale, and $\mathbf{v}_{l}$ is the vector with the photometric values. The database of the model features extracted from a model image $I_{m}$ is then denoted as $\mathcal{O}_{m}=\mathcal{O}\left(I_{m}, \Lambda_{o}\right)=\left\{\mathbf{f}_{l} \mid \mathbf{x}_{l} \in \mathcal{I}\left(I_{m}, \Lambda_{o}\right)\right\}$, where $\Lambda_{o}=\left(2^{\frac{k}{4}}\right)$, with $k=0, \ldots, 12$ representing the set of scales at which the image $I_{m}$ was processed and the set of interest points $\mathcal{I}\left(I_{m}, \Lambda_{o}\right)$ is defined as the set of positions in image $I_{m}$ selected at each scale in $\Lambda_{o}$ as interest points. Specifically, in this work, we study the local phase feature [8] and the scale-invariant feature transform (SIFT) feature [25]. The local phase feature is computed from the responses of the second derivative of a Gaussian and its Hilbert transform [19], which form a local complex representation that can be denoted by amplitude and phase. The interest points for the local phase feature are based on the multiscale Harris corner points [20], where the points presenting phase singularities [18] are filtered out [8]. The SIFT features [25] are computed using histograms of gradient values at several scales and the interest points are locations at the maxima and minima of a difference of Gaussian (DOG) function applied in scale space. Note that other types of local image features containing appearance and geometric information could also have been used in this work.

\subsection{Correspondence Set}

A correspondence set represents a data association between the set of model features $\mathcal{O}_{m}$ and a set of features $\mathcal{O}_{t}$ extracted from test image $I_{t}$. This set is denoted by

$$
\mathcal{N}_{m t}=\left\{\left(\mathbf{f}_{l}, \tilde{\mathbf{f}}_{1}\right) \mid \tilde{\mathbf{f}}_{l} \in \mathcal{O}_{t}, \mathbf{f}_{l} \in \mathcal{K}\left(\tilde{\mathbf{f}}_{1}, \mathcal{O}_{m}, \kappa_{\mathcal{N}}\right), s_{f}\left(\mathbf{f}_{l}, \tilde{\mathbf{f}}_{\mathrm{l}}\right)>\tau_{s}\right\},
$$


where the similarity function $s_{f}(.) \in[0,1]$ represents the similarity between two features $\left(s_{f}(.) \approx 1\right.$ means high similarity) and $\mathcal{K}($.$) is the set of the top- \kappa_{\mathcal{N}}$ correspondences between the test image feature $\tilde{\mathbf{f}}_{1} \in \mathcal{O}_{t}$ and the database of model features $\mathcal{O}_{m}$ in terms of the similarity function.

\section{Methods to Reject Mismatches}

In this section, we present our methods to reject mismatches from a correspondence set, where the key idea exploited is the use of semilocal constraints. In Section 4.1, we describe the grouping method based on pairwise relations between local image features and, in Section 4.2, we introduce our semilocal image feature.

\subsection{Grouping Based on Pairwise Relations}

One way of rejecting mismatches from the correspondence set is through a grouping stage. Typical grouping approaches for local features (for example, the Hough transform [25] and RANSAC [41]) rely on the global spatial configuration of features. Generally, these methods have become popular due to their efficiency and reasonably good performance for rejecting mismatches. However, a common property present in these approaches is the trade-off between the efficacy to reject mismatches and the robustness to large deviations from the chosen class of transformations. Since the class of transformations is usually globally rigid (for example, similarity or affine), any type of nonrigid deformation would cause these methods to reject correct matches and to break large sets of appropriate matches into several small-sized groups.

We propose a new grouping approach that aims at fixing these problems, with a time complexity comparable to the methods based on the global spatial configuration. Specifically, our grouping algorithm is designed to be robust to a broader class of deformations, which aims at reducing the number of groups, where each group has a higher percentage of correct matches and a higher number of correspondences. Our approach involves a connected component analysis (CCA) on an affinity matrix based on the pairwise relations.

\subsubsection{Pairwise Relations}

The pairwise geometric relations are composed of three measures between pairs of model features $\mathbf{f}_{l}, \mathbf{f}_{o} \in \mathcal{O}_{m}$ :

\begin{tabular}{|c|c|c|}
\hline scale & distance & heading \\
\hline $\mathcal{S}\left(\mathbf{f}_{l}, \mathbf{f}_{o}\right)=\frac{\left(\sigma_{l}-\sigma_{o}\right)}{\sqrt{\sigma_{l}^{2}+\sigma_{o}^{2}}}$ & $\mathcal{D}\left(\mathbf{f}_{l}, \mathbf{f}_{o}\right)=\frac{\left\|\mathbf{x}_{l}-\mathbf{x}_{o}\right\|}{\sqrt{\sigma_{l}^{2}+\sigma_{o}^{2}}}$ & $\mathcal{H}\left(\mathbf{f}_{l}, \mathbf{f}_{o}\right)=\Delta_{\theta}\left(\theta_{l}-\vartheta_{l o}\right)$, \\
\hline
\end{tabular}

where $\sigma_{k}$ is the scale of image feature $\mathbf{f}_{k}, \mathbf{x}_{k}$ is the image position of $\mathbf{f}_{k}, \Delta_{\theta}(.) \in(-\pi,+\pi]$ denotes the principal angle, $\theta_{k}$ is the main orientation of feature $\mathbf{f}_{k}$ for $k=l, o$, and $\vartheta_{l o}=\tan ^{-1}\left(\mathbf{x}_{l}-\mathbf{x}_{o}\right)$. The heading measurement considers the main orientation $\theta_{l}$ of feature vector $\mathbf{f}_{l}$ relative to the displacement between $\mathbf{x}_{l}$ and $\mathbf{x}_{o}$.

We can build the same pairwise relations between test image features $\tilde{\mathbf{f}}_{l}, \tilde{\mathbf{f}}_{\mathrm{o}} \in \mathcal{O}_{t}$ such that $\left(\mathbf{f}_{l}, \tilde{\mathbf{f}}_{l}\right),\left(\mathbf{f}_{o}, \tilde{\mathbf{f}}_{o}\right) \in \mathcal{N}_{m t}(1)$, thus forming $\mathcal{S}\left(\tilde{\mathbf{f}}_{1}, \tilde{\mathbf{f}}_{\mathrm{o}}\right), \mathcal{D}\left(\tilde{\mathbf{f}}_{1}, \tilde{\mathbf{f}}_{\mathrm{o}}\right)$, and $\mathcal{H}\left(\tilde{\mathbf{f}}_{1}, \tilde{\mathbf{f}}_{\mathrm{o}}\right)$. The pairwise semilocal spatial similarity is then based on

\begin{tabular}{|c|c|}
\hline scale & $\Delta \mathcal{S}_{l o}\left(\mathcal{N}_{m t}\right)=\mathcal{S}\left(\mathbf{f}_{l}, \mathbf{f}_{o}\right)-\mathcal{S}\left(\tilde{\mathbf{f}}_{1}, \tilde{\mathbf{f}}_{o}\right)$ \\
\hline distance & $\Delta \mathcal{D}_{l o}\left(\mathcal{N}_{m t}\right)=\mathcal{D}\left(\mathbf{f}_{l}, \mathbf{f}_{o}\right)-\mathcal{D}\left(\tilde{\mathbf{f}}_{l}, \tilde{\mathbf{f}}_{o}\right)$ \\
\hline heading & $\Delta \mathcal{H}_{l o}\left(\mathcal{N}_{m t}\right)=\mathcal{H}\left(\mathbf{f}_{l}, \mathbf{f}_{o}\right)-\mathcal{H}\left(\tilde{\mathbf{f}}_{l}, \tilde{\mathbf{f}}_{o}\right)$ \\
\hline
\end{tabular}

We define the similarity weight of the connection between $\tilde{\mathbf{f}}_{1}, \tilde{\mathbf{f}}_{\mathrm{o}} \in \mathcal{O}_{t}$ in the test image based on the connection of their respective correspondences in the model $\mathbf{f}_{l}, \mathbf{f}_{o} \in \mathcal{O}_{m}$ as follows:

$$
\begin{aligned}
& \mathbf{A}(l, o)= \\
& \delta_{m_{l} m_{o}} \pi_{l o, g} g\left(\left[\Delta \mathcal{D}_{l o}\left(\mathcal{N}_{m t}\right), \Delta \mathcal{H}_{l o}\left(\mathcal{N}_{m t}\right), \Delta \mathcal{S}_{l o}\left(\mathcal{N}_{m t}\right)\right]^{T} ; \Sigma_{\Delta}\right),
\end{aligned}
$$

where $m_{l}$ is the model index of feature $\mathbf{f}_{l}$ matched to deformed feature $\tilde{\mathbf{f}}_{\mathbf{l}}$ and, similarly, for $m_{o}$, and $\delta_{m_{l}, m_{o}}=1$ if $m_{l}=m_{o}$ and 0 otherwise. Also, the pairwise weight $\pi_{l o, g}$ is defined as

$$
\pi_{l o, g}=e^{-0.5 \frac{\mathcal{D}^{2}\left(\mathbf{f}_{l}, \mathbf{f}_{o}\right)}{\sigma_{\pi, g}^{2}}}
$$

where $\sigma_{\pi, g}=\frac{D_{M}}{L_{\text {pair }}}$, with $L_{\text {pair }}$ being a tuning variable, and $D_{M}$ is the maximum model diameter in pixels. Finally, $g($.) is the zero-mean unnormalized Gaussian function defined as $g(\mathbf{v} ; \Sigma)=e^{-\mathbf{v}^{T} \Sigma^{-1} \mathbf{v} / 2}$, where the covariance matrix $\Sigma_{\Delta}$ is a $3 \times 3$ diagonal matrix with distance, scale, and heading variances, namely, $\sigma_{d}^{2}, \sigma_{h}^{2}$, and $\sigma_{s}^{2}$, respectively, such that $\sigma_{h}^{2}$ and $\sigma_{s}^{2}$ are predefined constants, and $\sigma_{d}^{2}=\min \left(\kappa_{d i s t}\right.$, $\left.\max \left(p_{\text {dist }} \mathcal{D}\left(\mathbf{f}_{l}, \mathbf{f}_{o}\right), 0.1\right)\right)$ depends on the scaled original distance between model features $\mathbf{f}_{l}, \mathbf{f}_{o} \in \mathcal{O}_{m}$ (that is, points that are far from each other in the model have a proportionally larger standard error for their relative distances).

\subsubsection{Grouping Algorithm}

Given the correspondences $\mathcal{N}_{m t}$ (1) between the database of model features $\mathcal{O}_{m}$ and the set of test image features $\mathcal{O}_{t}$, we proceed as follows:

1. Build the affinity matrix based on the pairwise similarity measures $A(l, o)$ (see (4) and Step 1 in Fig. 1).

2. Perform a CCA. The strategy here is to select a weak threshold $\tau_{\mathrm{CCA}}$ and connect every pair of points $l$ and $o$ for which $\mathbf{A}(l, o) \geq \tau_{\mathrm{CCA}}$, thus forming $G$ connected clusters represented by the submatrix $\mathbf{A}_{g}$. We have then the subgroup of correspondences $\mathcal{L}_{g}\left(\mathcal{N}_{m t}\right) \subseteq$ $\mathcal{N}_{m t}$ composed of the features grouped in $\mathbf{A}_{g}$. Note that a specific cluster of correspondences can only belong to a single model $\mathcal{O}_{m}$ due to the term $\delta_{m_{l}, m_{o}}$ in (4) (see Step 2 in Fig. 1).

The complexity of this grouping algorithm is $O\left(\left|\mathcal{N}_{m t}\right|^{2}\right)$, where $\left|\mathcal{N}_{m t}\right|$ denotes the size of the correspondence set. Thus, a good strategy to keep the complexity of this algorithm manageable is to set $\tau_{s}$ at a high value and $\kappa_{\mathcal{N}}$ at a low value in (1) so that $\left|\mathcal{N}_{m t}\right|$ is reasonably small.

\subsection{Semilocal Image Features}

An intuitive method to improve the disambiguating power of local features is to group them in some predefined manner and use these groups as indexes to the model database [14]. Although several cues for clustering visual features have been proposed in [6], [24], we only exploit local feature proximity in this work. More specifically, we propose a method to verify the correctness of a given correspondence by using a variation of the shape context descriptor [4]. 


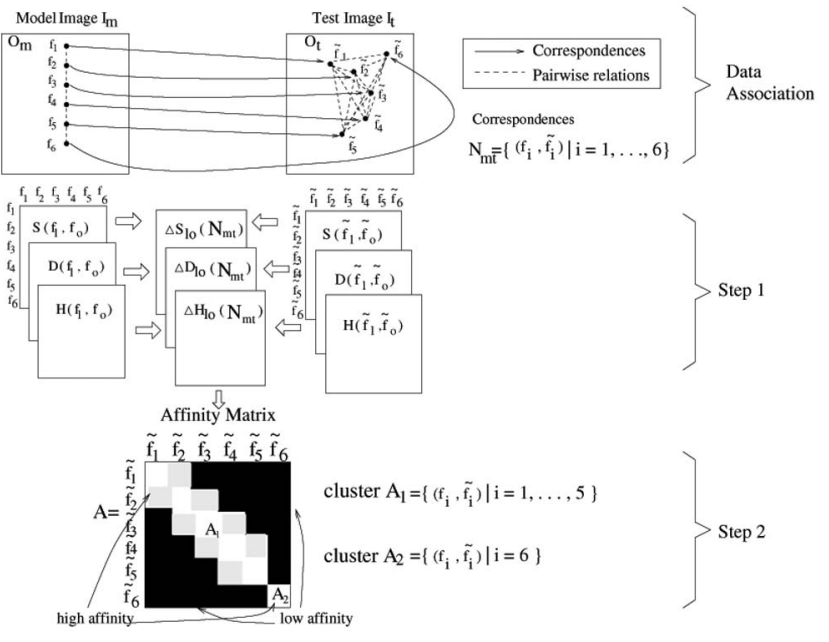

Fig. 1. Grouping based on pairwise relations. The data association consists of the matching model and test features based solely on the similarity of their feature values (see Section 3.1). Step 1 builds the pairwise similarity matrix, as described in Section 4.1.1 and Step 2 comprises the clustering algorithm based on CCA, as defined in Section 4.1.2. Notice that correspondences 1-5 are semilocally connected, whereas correspondence 6 is not. Therefore, two clusters are formed.

\subsubsection{Variation of Shape Context}

The shape context feature proposed in [4] is based on a logpolar space histogram, as shown in Fig. 2. Although shown to be useful in some recognition tasks, this image feature presents a few weaknesses in terms of robustness, which needed to be addressed in order to improve the discriminating power of typical local features. Assuming that we are augmenting the feature $\mathbf{f}_{l}$ and that $\mathbf{f}_{o}$ is a neighboring feature, the modifications made to the original shape context are listed as follows:

1. The robustness to nonrigid deformations is improved by weighting a vote in a specific histogram bin by

$$
w\left(\mathbf{f}_{l}, \mathbf{f}_{o}\right)=e^{\frac{-0.5 D^{2}\left(\mathbf{f}_{l} \mathbf{f}_{o}\right)}{L^{2}}},
$$

where $\mathcal{D}\left(\mathbf{f}_{l}, \mathbf{f}_{o}\right)$ is defined in (2), and $L=\frac{D_{M}}{L_{\mathrm{sc}}}$, with $L_{\mathrm{sc}}$ being a tuning variable, and $D_{M}$ is the maximum model diameter in pixels (in Fig. 2, darker cells in the histogram represent higher weight).

2. In order to reduce the boundary effects in the histogram, each neighboring feature votes for the two closest bins in each dimension (see in Fig. 2 that each vote spans four bins).

3. We make the shape context robust to rotation changes by rotating the histogram axis according to the main orientation of the feature.

4. The distance measures are scaled as in (2) in order to make them robust to scale changes.

The shape context similarity is computed using the $\mathcal{X}^{2}\left(h\left(\mathbf{f}_{l}\right), h\left(\tilde{\mathbf{f}}_{\mathbf{l}}\right)\right)$ test statistic defined in [4] as follows:

$$
\begin{aligned}
s_{h}\left(h\left(\mathbf{f}_{l}\right), h\left(\tilde{\mathbf{f}}_{l}\right)\right) & =1-\mathcal{X}^{2}\left(h\left(\mathbf{f}_{l}\right), h\left(\tilde{\mathbf{f}}_{l}\right)\right) \\
& =1-\frac{1}{2} \sum_{k=1}^{K} \frac{\left[h_{k}\left(\mathbf{f}_{l}\right)-h_{k}\left(\tilde{\mathbf{f}}_{l}\right)\right]^{2}}{h_{k}\left(\mathbf{f}_{l}\right)+h_{k}\left(\tilde{\mathbf{f}}_{l}\right)} \in[0,1],
\end{aligned}
$$

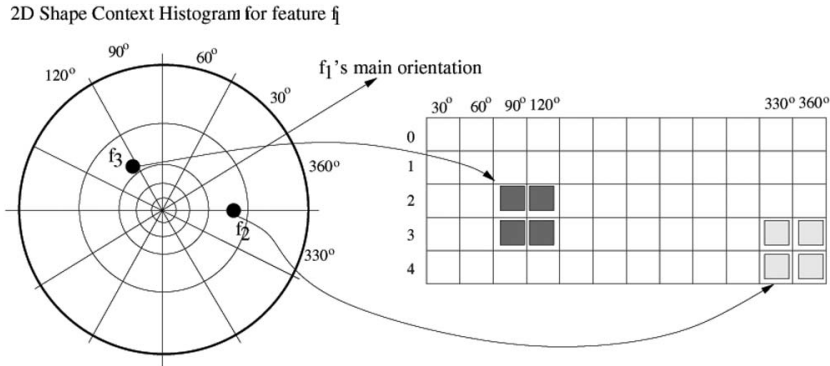

Fig. 2. Shape context of local feature $\mathbf{f}_{1}$. As in [4], we also use five bins for $\log$ (distance) and 12 bins for relative orientation. Note that we modify the original shape context method, as explained in Section 4.2.1.

where $h\left(\mathbf{f}_{l}\right)$ and $h\left(\tilde{\mathbf{f}}_{l}\right)$ are the $K$-bin normalized histograms of features $\mathbf{f}_{l}$ and $\tilde{\mathbf{f}}_{1}$, respectively. Therefore, given an initial set of correspondences $\mathcal{N}_{m t}(1)$ built using a feature similarity function $s_{f}($.$) , we select the features belonging to a common$ model that also have shape context similarity above some value $\tau_{c}$. This forms $G$ groups $\mathcal{L}_{g}\left(\mathcal{N}_{m t}\right)=\left\{\left(\tilde{f}_{l}, \tilde{\mathbf{f}}_{\mathbf{l}}\right) \mid\left(\mathbf{f}_{l}, \tilde{\mathbf{f}}_{\mathbf{l}}\right) \in\right.$ $\left.\mathcal{N}_{m t}, s_{h}\left(h\left(\mathbf{f}_{l}\right), h\left(\tilde{\mathbf{f}}_{\mathbf{f}}\right)\right)>\tau_{c}\right\}$, where $\forall\left(\mathbf{f}_{l}, \tilde{\mathbf{f}}_{l}\right),\left(\mathbf{f}_{o}, \mathbf{f}_{o}\right) \in \mathcal{L}_{g}\left(\mathcal{N}_{m t}\right)$, $m_{l}=m_{o}$ (that is, feature correspondences belonging to the same group $\mathcal{L}_{g}\left(\mathcal{N}_{m t}\right)$ must belong to the same model). Hence, $\bigcup_{g=1}^{G} \mathcal{L}_{g}\left(\mathcal{N}_{m t}\right) \subseteq \mathcal{N}_{m t}$. Note that this system is able to detect only one instance per model stored in the database, so the maximum number of groups formed equals the number of models stored in the database.

The performance improvement of this new semilocal feature is assessed using the quantitative evaluation, as described in Appendix A, which can be found at http:// computer.org/tpami/archives.htm. For these comparisons, we use the local phase features where the similarity function is denoted by (see [8] for details)

$$
s_{f}\left(\mathbf{f}_{l}, \tilde{\mathbf{f}}_{\mathrm{l}}\right)=\frac{\left|\mathbf{v}_{l} \cdot \tilde{\mathbf{v}}_{1}^{*}\right|}{1+\left|\mathbf{v}_{l}\right|\left|\tilde{\mathbf{v}}_{l}\right|},
$$

where $\mathbf{v}_{k}$ is a complex-valued vector, $\mathbf{v}_{k}^{*}$ represents its complex conjugate for $k=l, 1$, and - denotes dot product. We also use SIFT [25], where the similarity function is $s_{f}\left(\mathbf{f}_{l}, \tilde{\mathbf{f}}_{\mathrm{l}}\right)=\frac{1}{\left\|\mathbf{v}_{l}-\tilde{\mathbf{v}}_{1}\right\|}$. Finally, $L_{\mathrm{sc}}=100$ in (5).

We generate the receiver operating characteristic (ROC) curves by varying the feature similarity threshold $\tau_{s}$ and then evaluating the true positive (TP) and false positive (FP) by using the threshold values $\tau_{c} \in\{0,0.65,0.75,0.8,0.9\}$ for the shape context similarity function such that $s_{h}\left(h\left(\mathbf{f}_{l}\right), h\left(\tilde{\mathbf{f}}_{1}\right)\right)>$ $\tau_{c}$ (see (6)). Notice that when $\tau_{c}=0$, we are not using the shape context.

Fig. 3 shows the TP rates for an FP rate of 0.1 percent for the image deformations $d \in \mathcal{D F}$ described in Appendix B, which can be found at http://computer.org/tpami/archives.htm. Note that the size of the error bars in the graphs is large due to a combination of two things: 1) a small number of descriptors present in some of the test images (especially for the SIFT descriptor) and 2) a large number of cases where the $\mathrm{TP}$ rate is zero for an FP rate $=0.1$ percent. The correct matches and mismatches that are rejected from the correspondence set as $\tau_{c}$ increases (with $\mathrm{FP}=0.1$ percent) are shown in Fig. 4. The correct match rejection is computed as

$$
\frac{N_{i n}(0)-N_{i n}\left(\tau_{c}\right)}{N_{i n}(0)},
$$




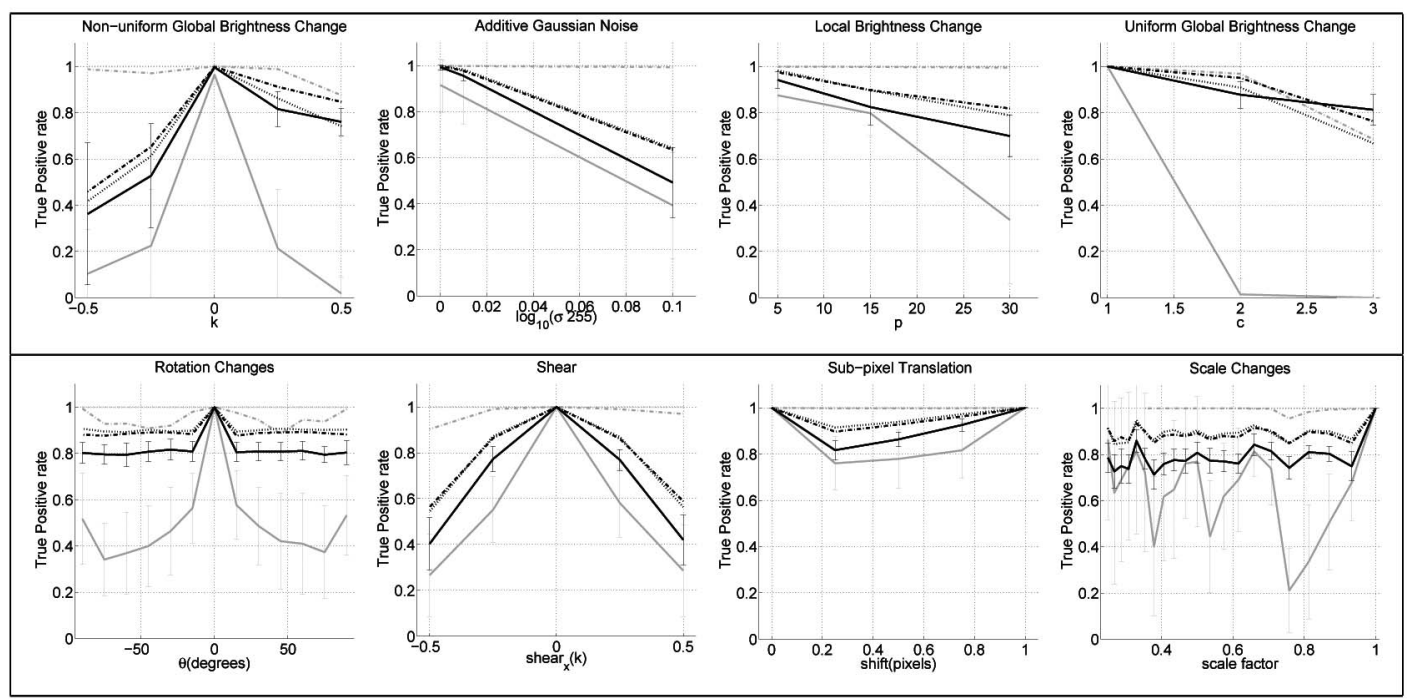

Fig. 3. The TP rate curves in terms of the image deformations $d \in \mathcal{D F}$ are obtained by holding the FP rate at 0.1 percent in the ROC curves generated by the evaluation experiment in Appendix A, which can be found at http://computer.org/tpami/archives.htm. Black curves are the phase local feature [8] without shape context (solid), with shape context such that $\tau_{c}=0.65$ (dashed), and $\tau_{c}=0.8$ (dotted). The gray curve shows the performance of SIFT [25] without shape context (solid), with shape context such that $\tau_{c}=0.65$ (dashed), and $\tau_{c}=0.8$ (dotted). Note that the error bars are omitted for the dashed and dotted curves for clarity but are of roughly the same size as the ones that we show.

where $N_{i n}\left(\tau_{c}\right)$ is the number of correct matches (see the computation of the TP rate above) for a given $\tau_{c}$, whereas the mismatches rejection is calculated as

$$
\frac{\left(N_{t o t}(0)-N_{\text {in }}(0)\right)-\left(N_{\text {tot }}\left(\tau_{c}\right)-N_{\text {in }}\left(\tau_{c}\right)\right)}{\left(N_{\text {tot }}(0)-N_{\text {in }}(0)\right)},
$$

where $N_{\text {tot }}\left(\tau_{c}\right)$ is the total number of features in the correspondence set for a given $\tau_{c}$. From these curves, it is clear that the use of shape context rejects many mismatches while keeping most of the correct matches in the correspondence set. It is interesting to notice in Figs. 3 and 4 that the use of shape context is more effective to remove mismatches in the correspondence sets of SIFT features than in the sets of local phase features. A possible reason for this is the combination of a relatively smaller number of SIFT features detected in an image and the robustness of the interest point detector DOG to the image deformations studied. It can also be seen in Fig. 3 that the local phase feature alone performs better than SIFT. This happens not only because the local phase information is robust to geometric transformations and brightness variations [18] but also because the relatively higher number of local phase descriptors per image (compared to the number of SIFT descriptors) increases the chances of a successful match in the deformed test image.

Similar to the pairwise clustering, the time complexity to build the semilocal feature is $O\left(\left|\mathcal{N}_{m t}\right|^{2}\right)$, where $\left|\mathcal{N}_{m t}\right|$
Phase Feature

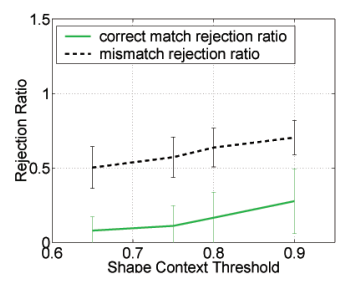

\section{SIFT}

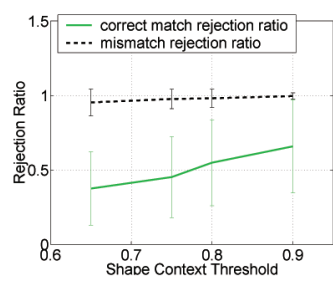

Fig. 4. Correct match and mismatch rejection ratios for our local phase feature and SIFT [25] by using shape context to reject mismatches. denotes the size of the correspondence set. Again, a good strategy to keep the complexity of this algorithm manageable is to set $\tau_{s}$ at a relatively high value and $\kappa_{\mathcal{N}}$ at a low value in (1) so that $\left|\mathcal{N}_{m t}\right|$ is reasonably low.

\subsection{Performance Evaluation}

A comparison between our mismatch rejection methods described above and the generalized the Hough transform is provided next. The reason for comparing our methods against the Hough transform resides in its attractive properties, which include 1) low time complexity, 2) reasonably high accuracy, and 3) wide availability and acceptance. We intend to show that our methods prune the initial correspondence set more accurately than the Hough transform, generating groups with a higher rate of correct matches in terms of not only nonrigid but also rigid transformations. We also illustrate that the efficiency of our method is comparable to the one presented by the Hough transform for typical matching problems.

In the experiments below, we used the phase-based local feature for the model representation, with the feature similarity defined by (7). For the pairwise clustering scheme, we assumed the following values for the constants in (4): the standard deviation of heading, scale, and distance are, respectively, $\sigma_{h}^{2}=0.2, \quad \sigma_{s}^{2}=0.2, \quad \sigma_{d}^{2}=\min \left(\kappa_{\text {dist }}, \max \left(p_{\text {dist }} \mathcal{D}\right.\right.$ $\left.\left.\left(\mathbf{f}_{l}, \mathbf{f}_{o}\right), 0.1\right)\right)$, with $\kappa_{\text {dist }}=2$ and $p_{\text {dist }}=0.2$, and $L_{\text {pair }}=5$ for the computation of pairwise weight (4). In order to generate the graphs below, we vary the parameter $\tau_{\mathrm{CCA}}=k / 10$ for $k=\{1,2, \cdots, 9\}$, which is the threshold for the CCA algorithm described in Section 4.1. For the semilocal feature, the parameter $L_{\mathrm{sc}}=100$ is used in the computation of (5). We vary the threshold for the shape context similarity between corresponding features in order to generate the graphs by using $\tau_{c}=k / 10$ for $k=\{4,4.5,5, \ldots, 9\}$. Therefore, this mismatch rejection method discards any correspondence $\left(\mathbf{f}_{l}, \tilde{\mathbf{f}}_{l}\right) \in \mathcal{N}_{t}$ that $s_{h}\left(h\left(\mathbf{f}_{l}\right), h\left(\tilde{\mathbf{f}}_{l}\right)\right)<\tau_{c}$ (see (6)).

The Hough clustering algorithm builds a transform space (for example, similarity or affine), and using each element of $\mathcal{N}_{m t}$ in (1) as a point in this space, it finds groups of points that 

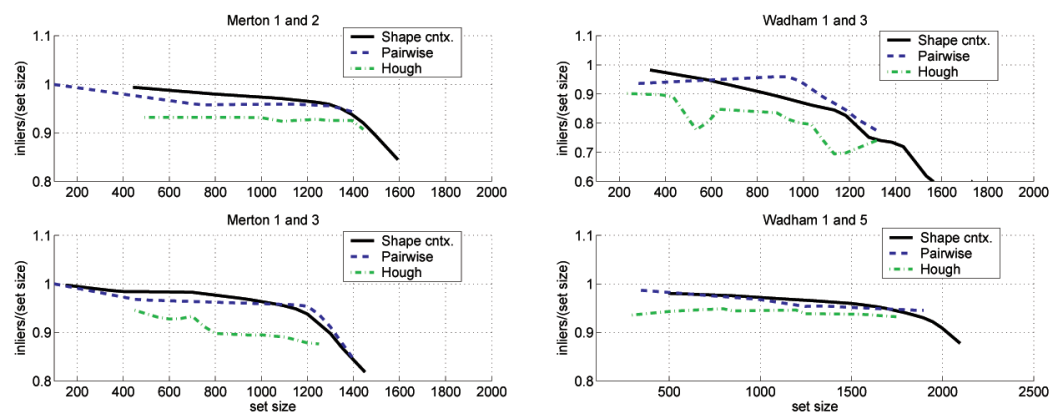

Fig. 5. Quantitative comparisons between our mismatch rejection methods and Hough transform for rigid transformations. The comparisons show the proportion of correct matches from the correspondence sets of varying size provided by each of the mismatch rejection methods.
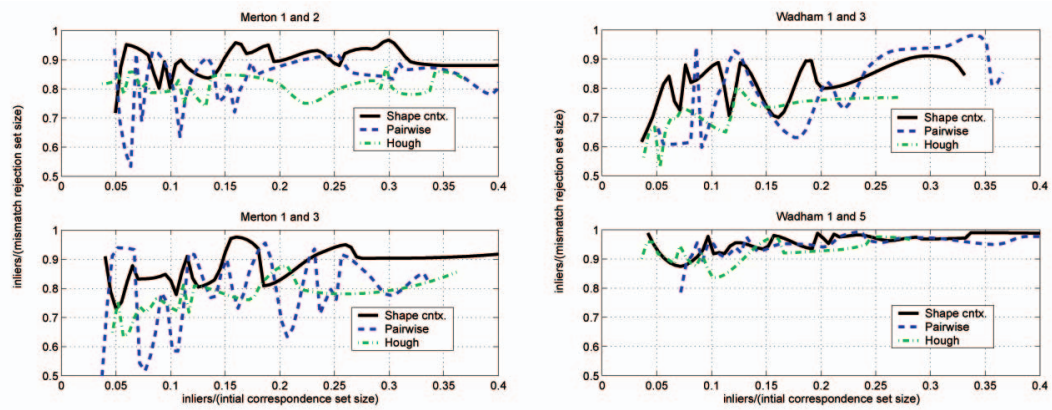

Fig. 6. Quantitative comparisons that show the proportion of correct matches as a function of the percentage of inliers present in the initial correspondence set built from the feature similarity search.

move coherently according to the transformation being modeled. For the experiments in this section, we use a space of similarity transform in the Hough clustering algorithm with the following bin sizes for translation: $\{0.3,0.15,0.05\}$ times $D_{M}$ (that is, the maximum model diameter). For rotation, the bin sizes studied are $\{30,15,5\}$ degrees. The bin sizes above are varied in order to produce the results for the experiments in the next section. We did not vary the scale bin sizes since the examples considered do not present much variability in terms of scale. Instead, the histogram for scale changes has the following fixed bin values: $[0.125,0.25,0.5,1$, $2,4,8,16]$. Finally, each hypothesis is hashed into the two closest bins in each dimension in order to reduce the boundary effects. Also, in order to avoid a high number of groups, we run a nonmaximum suppression when searching for the local maxima in this space. Note that the complexity of the Hough transform is simply the number of bins in this transformation space.

\subsubsection{Rigid Transformation}

In order to show the effectiveness of our approaches with respect to rigid transformation, we consider the wide baseline matching problem. Using the set provided by each mismatch rejection method, we compute the $\mathbf{F}$ matrix as presented in [21] by using RANSAC [41]. We are interested in computing the proportion of inliers, given the size of this set. An inlier is considered to be a feature that lies within four pixels (approximately, the spatial resolution of the local features used) of the epipolar lines computed from the $\mathbf{F}$ matrix. For this experiment, we used two sequences available from Oxford's Visual Geometry Group's Web page, namely, Wadham and Merton College sequences (see Figs. 19 and 20). In Fig. 5, we present the graphs of each matching. Note that the proportion of inliers for the correspondence sets of the same size is, for the cases studied, always higher for our methods than for Hough. These results show that for the correspondence sets containing on the order of 1,000 matches, there are around 90 percent to 95 percent of inliers. This means that the point prediction estimates might be affected by the remaining 5 percent to 10 percent mismatches. In Section 5, we propose a method to eliminate the remaining mismatches. Fig. 6 shows the robustness of each mismatch rejection method to high percentages of mismatches present in the initial correspondence set (the variation of the correspondence set size is obtained by varying the threshold in (1)). Notice that the semilocal feature presents the best robustness, since its performance is relatively stable even with the presence of a high percentage of mismatches, whereas both the pairwise clustering and Hough start presenting an unstable behavior when the initial proportion of correct matches falls below 15 percent.

For the experiments in this section, the number of operations carried out by the pairwise grouping and the semilocal feature algorithms is about $10^{6}$, which is proportional to $\left|\mathcal{N}_{m t}\right|^{2}$. Moreover, the number of operations of the Hough transform varies between $10^{5}$ and $10^{7}$, depending on the number of bins used in the transformation space.

\subsubsection{Nonrigid Deformation}

Two comparisons are presented in Figs. 7 and 8, where, for the pairwise clustering and the Hough transform, only the group that clustered the highest number of features is shown in each case. Note that, for the case of the semilocal feature, only one group per correspondence set can be formed, and this is the group shown in the experiments. Fig. 7 shows the results of our mismatch rejection methods proposed here and of the Hough transform, where the model is an object composed of a string built with soda cans. 


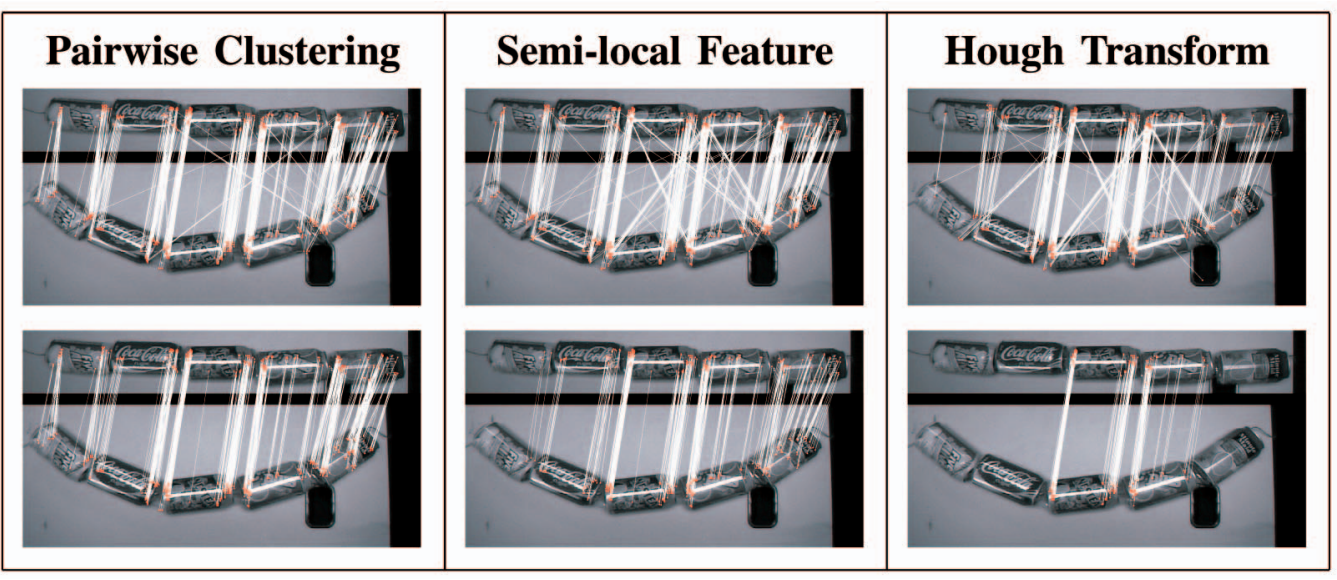

Fig. 7. Comparison between our mismatch rejection methods and Hough clustering for nonrigid deformation. The lines represent the feature correspondences that were grouped together by the respective method between the test image on the bottom and the model image on the top. The first row shows the results where the parameters of each method were set to be extremely tolerant to mismatches, whereas the second row shows the results where the parameters were set such that the group formed had the highest number of correspondences without any visible mismatch.

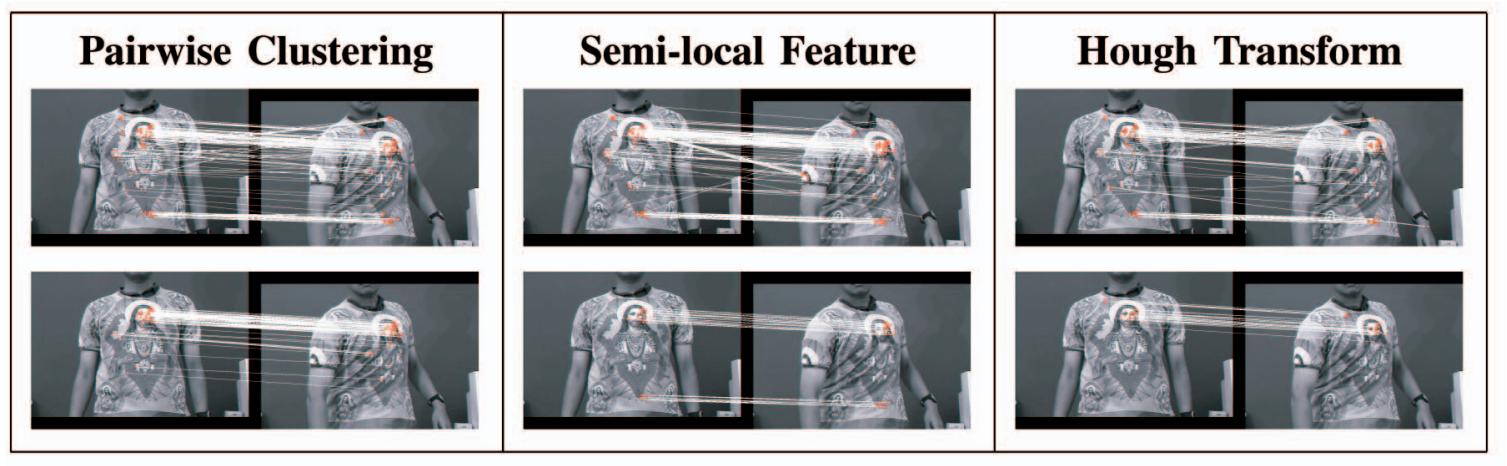

Fig. 8. Second comparison between our mismatch rejection algorithms and the Hough transform. Please refer to the caption of Fig. 7 for details.

For each method, two results are shown. In the first row, the parameters of each method are set to be extremely tolerant to mismatches, whereas the second row depicts the case where each method produces the largest correspondence set without any visually detectable mismatch. Notice that the Hough transform only matches a piece of the object that suffered a deformation close to a rigid transformation when its parameters are set to be robust to mismatches, whereas our methods tend to be more robust to nonrigid deformations even when they are very resistant to mismatches.

Finally, in the experiments above, the number of operations carried out by the pairwise grouping and the semilocal feature algorithms is around $10^{6}$, whereas that of the Hough transform varies between $10^{5}$ and $10^{7}$.

\subsubsection{Discussion}

Although both methods are shown to be effective at reducing the mismatches in correspondence sets, each one has advantages and disadvantages. The grouping based on pairwise relations shows a slightly higher robustness to nonrigid deformations, but it needs neighboring model points to be neighbors in the test image, which means that a large gap of neighboring matches in the correspondence set can potentially break the initial group into subgroups. One advantage of the semilocal method is its high robustness to mismatches in the correspondence sets, as depicted in Fig. 6. Another advantage of the semilocal feature is in terms of efficiency, where the computation of the shape feature can be performed in parallel to that of the local feature after the location and orientation of the interest points are determined, but the fact that it can form only one group per model may represent a problem in recognition tasks involving the detection of several instances of a model in a test image.

\section{Geometric Predictions}

The mismatch rejection methods presented in Sections 4.1 and 4.2 can be made arbitrarily robust to mismatches by varying the thresholds $\tau_{\mathrm{CCA}}$ in (4) and $\tau_{c}$ in (6). Generally, it is desirable to be tolerant at this stage and let the next stages in the system do the fine-tuning by rejecting mismatches that remained in the correspondence set. The main reason for letting the system accept a few mismatches at this first stage is to make it less prone to false negatives. Moreover, once we a have a correspondence set relatively free of mismatches, the system has to determine whether this set represents an instance of a model. Therefore, the geometric predictions that we present now have two objectives: 1) further reject mismatches from the correspondence sets and 2) provide a measure of the likelihood of model presence in the correspondence set.

Consider again the set of correspondences $\mathcal{N}_{m t}$ defined in (1) between the model features $\mathcal{O}_{m}$ and the test image features $\mathcal{O}_{t}$. The idea is to predict $\tilde{\mathbf{x}}_{\mathrm{k}}, \tilde{\theta}_{\mathrm{k}}$, and $\tilde{\sigma}_{\mathrm{k}}$ for each test image feature $\tilde{\mathrm{f}}_{\mathrm{k}} \in \mathcal{O}_{t}$ that has a correspondence in $\mathcal{N}_{m t}$ and compare those predicted values with the actual values of 


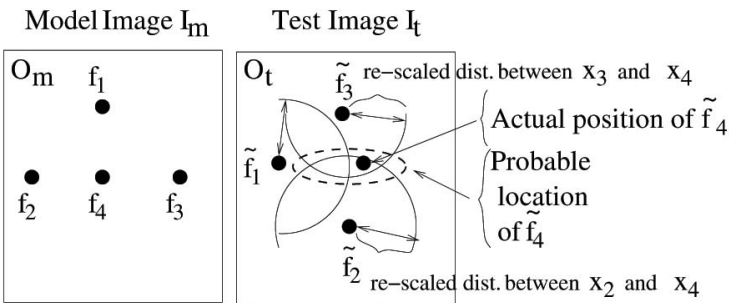

Fig. 9. Example of position prediction. Given the set of model features $\left\{\mathbf{f}_{l}\right\}_{l \in\{1,2,3,4\}}$, suppose we want to estimate the position of test image feature $\tilde{\mathbf{f}}_{4}$. The probable location of the feature (represented by a dotted ellipsoid) is based on a Gaussian distribution computed using the position of the correspondences in the test and model images and the pairwise variances $\sigma_{\mathcal{D}}^{2}\left(\mathbf{f}_{l}, \mathbf{f}_{o}\right)$ estimated in the learning stage.

the feature $\tilde{f}_{\mathrm{k}}$. This comparison is then used to measure the likelihood of the presence of $\tilde{f}_{\mathrm{k}}$ assuming the model presence. In general, note that the following relations are true if the correspondence is correct:

$$
\begin{aligned}
& \tilde{\mathbf{n}}_{\mathrm{lo}}^{T}\left(\tilde{\mathbf{x}}_{1}-\tilde{\mathbf{x}}_{\mathrm{o}}\right) \approx\left\|\mathbf{x}_{l}-\mathbf{x}_{o}\right\|, \text { where } \tilde{\mathbf{n}}_{\mathrm{lo}}=\frac{\tilde{\mathbf{x}}_{1}-\tilde{\mathbf{x}}_{o}}{\left\|\tilde{\mathbf{x}}_{1}-\tilde{\mathbf{x}}_{\mathrm{o}}\right\|}, \\
& \tilde{\theta}_{1}-\tilde{\vartheta}_{\mathrm{lo}} \approx \theta_{l}-\vartheta_{l o}, \\
& \frac{\tilde{\sigma}_{1}-\tilde{\sigma}_{\mathrm{o}}}{\tilde{\sigma}_{\mathrm{o}}} \approx \frac{\sigma_{l}-\sigma_{o}}{\sigma_{o}} .
\end{aligned}
$$

Let us consider the position prediction first. Assuming that the observed position $\tilde{\mathbf{x}}_{1}$ is affected by an additive Gaussian noise, we have

$$
\pi_{l o, p} \tilde{\mathbf{n}}_{\mathrm{lo}}^{T} \tilde{\mathbf{x}}_{l}=\pi_{l o, p}\left\|\mathbf{x}_{l}-\mathbf{x}_{o}\right\|+\pi_{l o, p} \tilde{\mathbf{n}}_{\mathrm{lo}}^{T} \tilde{\mathbf{x}}_{\mathrm{o}}+\pi_{l o, p} r_{\mathcal{D}}\left(\mathbf{f}_{l}, \mathbf{f}_{o}\right)
$$

for all $\left(\mathbf{f}_{o}, \tilde{\mathbf{f}}_{0}\right) \in \mathcal{N}_{m t}-\left(\mathbf{f}_{l}, \tilde{\mathbf{f}}_{1}\right)$, where $r_{\mathcal{D}}($.$) is a Gaussian$ noise with zero mean and variance $\sigma_{\mathcal{D}}^{2}\left(\mathbf{f}_{l}, \mathbf{f}_{o}\right)$, which is defined later in Section 6.2. Here,

$$
\pi_{l o, p}=e^{-0.5 \frac{\mathcal{D}\left(\mathfrak{f}_{l}, \mathbf{f}_{o}\right)}{\sigma_{\pi, p}}}
$$

is the pairwise weight, meaning that the neighboring points to $\mathbf{f}_{l}$ within a range of roughly $\sigma_{\pi, p}$ pixels have a higher weight in predicting the position of the test feature $\tilde{\mathbf{f}}_{1}$ than neighboring points that are farther away. We set the value of $\sigma_{\pi, p}$ as a fraction of the model diameter in pixels. Equation (9) can be rewritten as

$$
\Pi \mathbf{K}^{T} \tilde{\mathbf{x}}_{1}=\Pi \mathbf{b}+\Pi \mathbf{r}_{\mathcal{D}},
$$

where $\mathbf{K} \in \Re^{2 \times N-1}$ is a matrix with the vectors $\tilde{\mathbf{n}}_{\mathrm{lo}} \in \Re^{2 \times 1}$ in its columns, with $N$ being the number of correspondences in $\mathcal{N}_{m t}, \Pi \in \Re^{N-1 \times N-1}$ is a diagonal matrix with the values $\pi_{l o, p}$ for all $o \neq l, \mathbf{b} \in \Re^{N-1 \times 1}$ with $\mathbf{b}=\left\|\mathbf{x}_{l}-\mathbf{x}_{o}\right\|+\mathbf{n}_{l o}^{T} \tilde{\mathbf{x}}_{o}$ for all $o \neq l$, and $\mathbf{r}_{\mathcal{D}} \in \Re^{N-1 \times 1}$ is the vector with the Gaussian noise mentioned above. From (10), we have

$$
\tilde{\mathbf{x}}_{\mathrm{l}}=\mathbf{B b}+\mathbf{B} \mathbf{r}_{\mathcal{D}}
$$

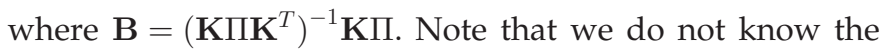
specific values of $r_{\mathcal{D}}($.$) but only their distribution, so we$ approximate the position $\tilde{\mathbf{x}}_{1}$ by the following prediction (see Fig. 9):

$$
\tilde{\mathbf{x}}_{1}^{*}=E\left[\tilde{x}_{1}\right]=\mathbf{B b}
$$

In order to compute the similarity between the observed position $\tilde{\mathbf{x}}_{1}$ and its prediction $\tilde{\mathbf{x}}_{1}^{*}$, we have to compute the position covariance as follows:

$$
\begin{aligned}
\Sigma_{\mathcal{D}}\left(\tilde{\mathbf{f}}_{1}\right) & =E\left[\left(\tilde{\mathbf{x}}_{1}-E\left[\tilde{\mathbf{x}}_{1}\right]\right)\left(\tilde{\mathbf{x}}_{1}-E\left[\tilde{\mathbf{x}}_{1}\right]\right)^{T}\right] \\
& =E\left[\mathbf{B r}_{\mathcal{D}} \mathbf{r}_{\mathcal{D}}^{T} \mathbf{B}^{T}\right]=\operatorname{B} \operatorname{diag}\left(\sigma_{\mathcal{D}}^{2}\left(\mathbf{f}_{l}, \mathbf{f}_{o}\right)\right) \mathbf{B}^{T}
\end{aligned}
$$

where $\sigma_{\mathcal{D}}^{2}\left(\mathbf{f}_{l}, \mathbf{f}_{o}\right)$ is assumed to be independent for all $o \neq l$. Finally, the similarity between $\tilde{\mathbf{x}}_{1}$ and $\tilde{\mathbf{x}}_{1}^{*}$ is computed as $\mathcal{G}\left(\tilde{\mathbf{x}}_{1}-\tilde{\mathbf{x}}_{1}^{*} ; \Sigma_{\mathcal{D}}\left(\tilde{\mathbf{f}}_{1}\right)\right)$, where $\mathcal{G}($.$) is the normalized zero-mean$ Gaussian function.

Following the same reasoning, the similarity between $\tilde{\theta}_{1}$ and $\tilde{\theta}_{1}^{*}$ is defined as $\mathcal{G}\left(\tilde{\theta}_{1}-\tilde{\theta}_{1}^{*} ; \sigma_{\mathcal{H}}^{2}\left(\tilde{\mathbf{f}}_{1}\right)\right)$, with $\mathcal{G}($.$) being, again,$ the normalized zero-mean Gaussian function, and

$$
\sigma_{\mathcal{H}}^{2}\left(\tilde{\mathbf{f}}_{\mathrm{l}}\right)=\left(\frac{1}{\sum_{o \neq l} \pi_{l o, p}}\right)^{2}\left(\sum_{o \neq l} \pi_{l o, p}^{2} \sigma_{\mathcal{H}}^{2}\left(\mathbf{f}_{l}, \mathbf{f}_{o}\right)\right),
$$

where $\sigma_{\mathcal{H}}^{2}\left(\mathbf{f}_{l}, \mathbf{f}_{o}\right)$ is defined as in Section 6.2. Finally, the similarity between $\tilde{\sigma}_{1}$ and $\tilde{\sigma}_{1}^{*}$ is computed as $\mathcal{G}\left(\tilde{\sigma}_{1}-\tilde{\sigma}_{1}^{*} ; \sigma_{\mathcal{S}}^{2}\left(\tilde{\mathbf{f}}_{1}\right)\right)$, with $\mathcal{G}($.) being the normalized zero-mean Gaussian function, and

$$
\sigma_{\mathcal{S}}^{2}\left(\tilde{\mathbf{f}}_{\mathbf{l}}\right)=\left(\frac{1}{\sum_{o \neq l} \pi_{l o, p}}\right)^{2}\left(\sum_{o \neq l} \pi_{l o, p}^{2} \sigma_{\mathcal{S}}^{2}\left(\mathbf{f}_{l}, \mathbf{f}_{o}\right)\right),
$$

where $\sigma_{\mathcal{S}}^{2}\left(\mathbf{f}_{l}, \mathbf{f}_{o}\right)$ is also defined as in Section 6.2.

Therefore, the similarity between the predicted and observed positions, main orientation, and scale is computed in just one step as follows:

$$
p\left(\mathbf{f}_{l}, \tilde{\mathbf{f}}_{l}\right)=\mathcal{G}\left(\left[\tilde{\mathbf{x}}_{1}, \tilde{\theta}_{l}, \tilde{\sigma}_{1}\right]-\left[\tilde{\mathbf{x}}_{1}^{*}, \tilde{\theta}_{1}^{*}, \tilde{\sigma}_{1}^{*}\right] ; \Sigma_{t}\right),
$$

where $\mathcal{G}($.$) is the normalized Gaussian function with zero$ mean, and $\Sigma_{t}=\operatorname{diag}\left(\Sigma_{\mathcal{D}}\left(\tilde{\mathbf{f}}_{1}\right), \sigma_{\mathcal{H}}^{2}\left(\tilde{\mathbf{f}}_{1}\right), \sigma_{\mathcal{S}}^{2}\left(\tilde{\mathbf{f}}_{1}\right)\right)$.

The likelihood of the correspondence between $\mathbf{f}_{l}$ and $\tilde{\mathbf{f}}_{1}$, represented by $p($.$) in (14), is used for two goals. The first is to$ form the final set of correspondences by thresholding $p($.) and forming the set $\tilde{\mathcal{L}}_{g}\left(\mathcal{N}_{m t}\right)=\left\{\left(\mathbf{f}_{l}, \tilde{\mathbf{f}}_{\mathrm{f}}\right) \mid\left(\mathbf{f}_{l}, \tilde{\mathbf{f}}_{\mathrm{l}}\right) \in \mathcal{L}_{g}\left(\mathcal{N}_{m t}\right)\right.$, $\left.p\left(\mathbf{f}_{l}, \tilde{\mathbf{f}}_{1}\right)>\tau_{p}\right\}$. The second goal is to use the value provided by $p($.$) to determine the likelihood of the correspondence$ between $\mathbf{f}_{l}$ and $\tilde{\mathbf{f}}_{1}$. The time complexity of this algorithm is, like the mismatch rejection methods above, $O\left(\left|\mathcal{N}_{m t}\right|^{2}\right)$, so it does not deteriorate the complexity of the system.

\subsection{Performance Evaluation}

In this section, we demonstrate the efficacy of the geometric prediction algorithm for the task of rejecting the remaining mismatches left by the mismatch rejection methods presented in Section 4.

The geometric prediction has two parameters to set. The first is the weight that a feature $\mathbf{f}_{o}$ has in predicting the position, orientation, and scale of a feature $\mathbf{f}_{l}$. We use

$$
\pi_{l o, p}=e^{\frac{-0.5 D^{2}\left(\mathbf{f}_{l}, \mathbf{f}_{o}\right)}{\sigma_{\pi, p}^{2}}},
$$

where we set $\sigma_{\pi, p}=\frac{D_{M}}{10}$. The other parameter is the correct match threshold $\tau_{p}$, which is set at $10^{-16}$.

1. Notice that we intentionally gave the same name for the sets of hypotheses to be verified $\tilde{\mathcal{L}_{g}}\left(\mathcal{N}_{m t}\right)$ built from both mismatch rejection methods (that is, semilocal features and grouping based on pairwise relations). 

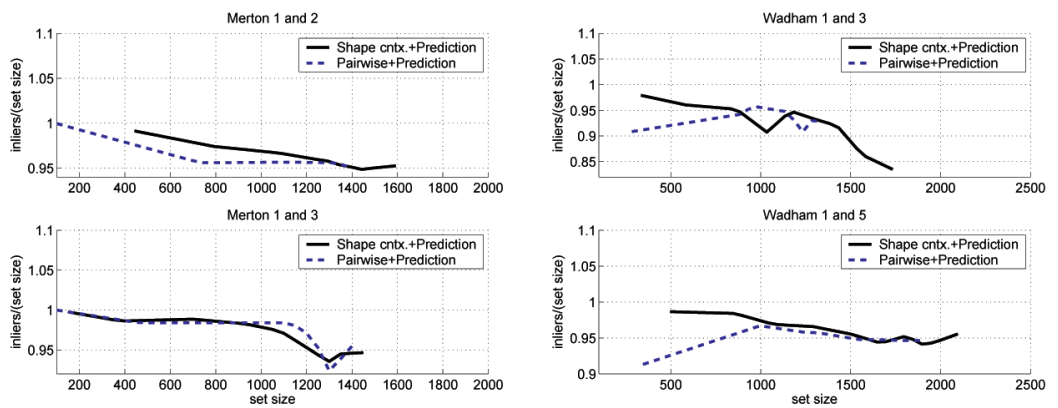

Fig. 10. Percentage of correct matches versus the correspondence set after geometric prediction. This graph represents an extension of the graphs in Fig. 5, but here, the geometric prediction filters out mismatches from the group formed by the respective mismatch rejection method. Note that the vertical scale is slightly different from that in Fig. 5.
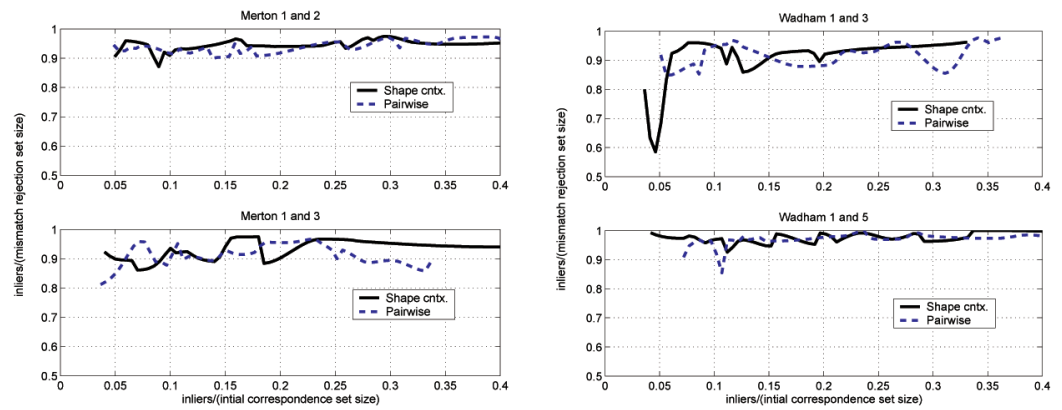

Fig. 11. Quantitative comparisons that show the proportion of correct matches as a function of the percentage of inliers present in the initial correspondence set. This graph represents an extension of the graphs in Fig. 6, but here, the geometric prediction filters out mismatches from the group formed by the respective mismatch rejection method.

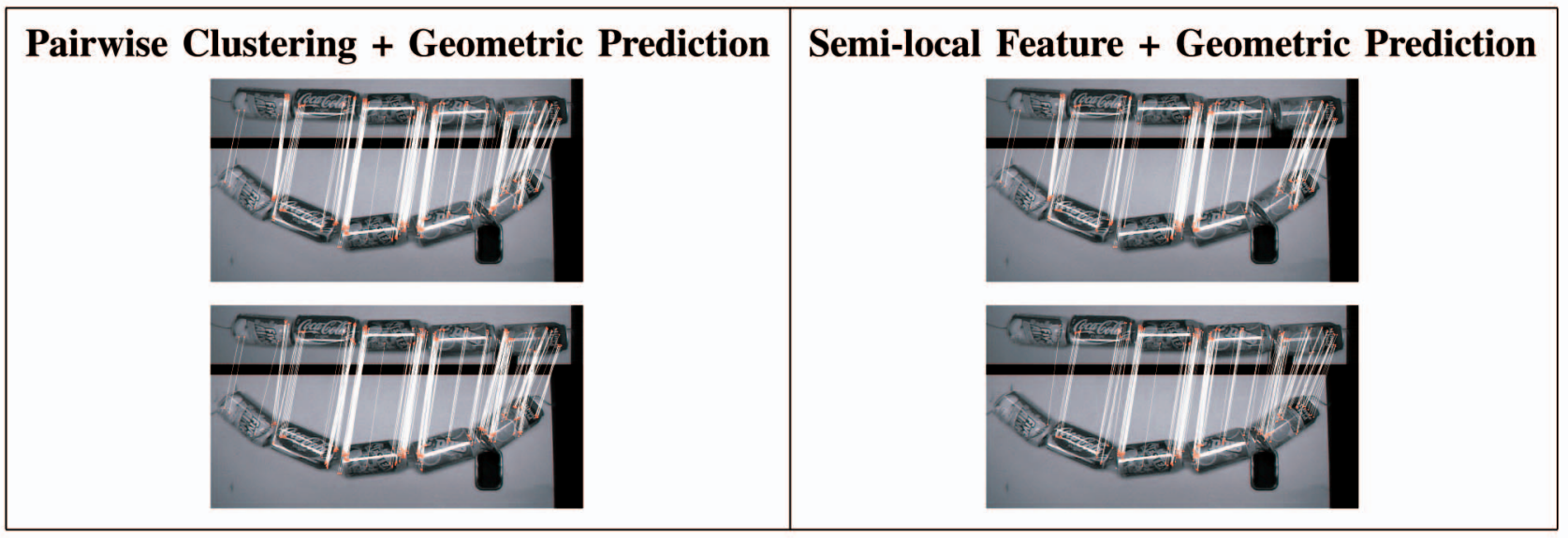

Fig. 12. Correspondence set after the geometric prediction method has filtered out the mismatches present in the groups in Fig. 7.

\subsubsection{Rigid Deformation}

The experimental setup introduced in Section 4.3 .1 is used here, and we show the results in the final correspondence set after the geometric prediction rejected the remaining outliers from the groups formed by both mismatch rejection methods. Fig. 10 shows the inlier percentage versus the correspondence set size for the respective graphs in Fig. 5. Note that the main difference is that the inlier percentage rarely falls below 90 percent to 95 percent even for large correspondence sets. Also, Fig. 11 illustrates the consistent robustness of the geometric prediction combined with the mismatch rejection methods to extremely high percentage of mismatches in the initial correspondence set. Even in cases with less than 10 percent of initial correct matches, both methods return a final correspondence set with generally more than 90 percent of inliers.

\subsubsection{Nonrigid Deformation}

We extend the experiment presented in Section 4.3.2, where the geometric prediction is used to reject the mismatches from the groups built by both mismatch rejection methods. Fig. 12 shows the results from the geometric prediction on the groups in Fig. 7, whereas Fig. 13 presents the final correspondence sets in Fig. 8.

\section{Probabilistic Formulation for Verification}

In this section, we introduce the probabilistic formulation for the hypothesis verification stage, which is based on [31], but we make somewhat less restrictive assumptions that may improve the verification performance.

The problem of constructing a probabilistic method for the verification of hypotheses has been intensively studied lately. 


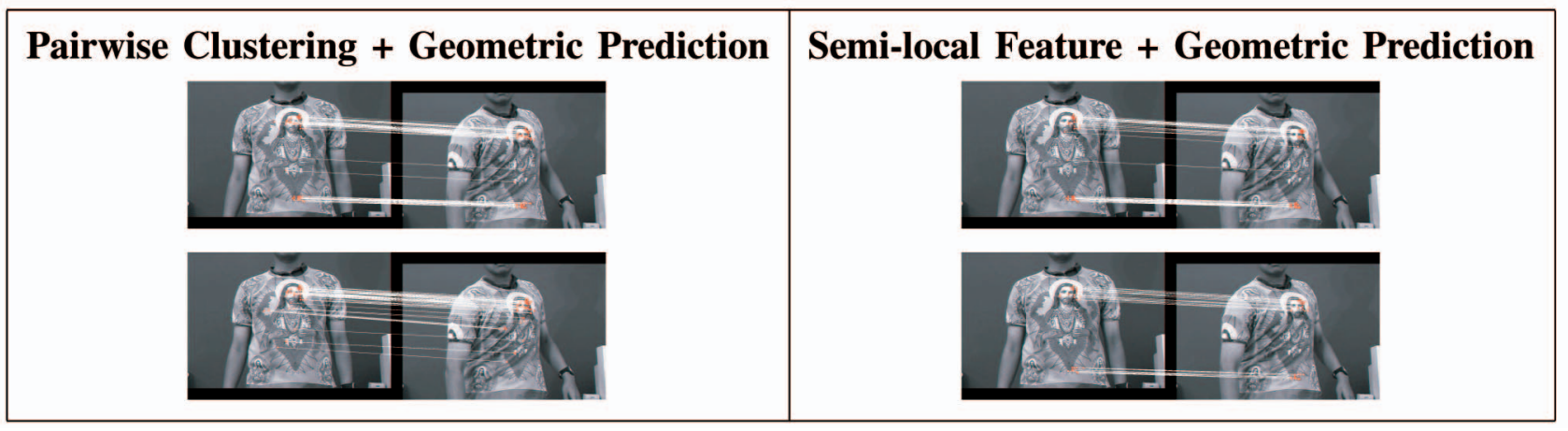

Fig. 13. Correspondence set after the geometric prediction algorithm has filtered out the remaining mismatches present in the groups in Fig. 8.

Similar probabilistic verification methods to recognize limited categories of objects are presented in [1], [2], [15], [16], [43], where the systems generally work with a small set of parts (substantially fewer than 100 parts). It is worth noting that among the papers cited before, only the work described in [1] uses a flexible spatial coherence based on pairwise relations for the verification. Systems more closely related to ours are described in [26], [31], [34]. Lowe [26] relies on a probabilistic verification that takes into account the global shape of the model and the information about the distinctiveness of the model as a whole. Schmid [34] describes a probabilistic verification that uses semilocal coherence, where a learning approach to estimate the feature appearance variation is described. However, it is likely that this system suffers from the presence of mismatches in large hypothesis sets.

In order to assess the hypothesis that a particular object is present in a test image, we propose a probabilistic formulation framework that involves the feature correspondences and the semilocal spatial configuration similarities. Assuming that $\mathcal{O}_{m}$ represents the hypothesis that an instance of the model $m$ is present in the test image, $\mathcal{E}$ is a set of correspondences, and $T$ represents the global geometric configuration of features (that is, their position $\mathbf{x}$, scale $\sigma$, and main orientation $\theta$ ). We define the posterior $P\left(\mathcal{O}_{m} \mid \mathcal{E}, T\right)$ as (using the Bayes rule):

$$
P\left(\mathcal{O}_{m} \mid \mathcal{E}, T\right)=\frac{P\left(\mathcal{E} \mid T, \mathcal{O}_{m}\right) P\left(T \mid \mathcal{O}_{m}\right) P\left(\mathcal{O}_{m}\right)}{\sum_{\mathcal{O}=\mathcal{O}_{m}, \neg \mathcal{O}_{m}} P(\mathcal{E} \mid T, \mathcal{O}) P(T \mid \mathcal{O}) P(\mathcal{O})}
$$

In [31], three assumptions are made:

1. $P(\mathcal{E}, T)=P(\mathcal{E}) P(T)$, that is, the correspondences are independent of their global geometrical configuration;

2. $P\left(T \mid \mathcal{O}_{m}\right)=P(T)$, which means that the global configuration is conditionally independent of the hypothesized model; and

3. $\frac{P\left(\mathcal{E} \mid T, \mathcal{O}_{m}\right)}{P(\mathcal{E})}=\prod_{i} \frac{P\left(e_{i} \mid T, \mathcal{O}_{m}\right)}{P\left(e_{i}\right)}$, where $e_{i} \mathrm{~S}$ are the individual elements of set $\mathcal{E}$.

On the other hand, we have two assumptions:

1. $P\left(T \mid \mathcal{O}_{m}\right)=P\left(T \mid \neg \mathcal{O}_{m}\right)=P(T)$, or the global geometrical configuration can be assumed to be conditionally independent of the hypothesized model.

2. $P\left(\mathcal{E} \mid T, \mathcal{O}_{m}\right)=\prod_{i} P\left(e_{i} \mid T, \mathcal{O}_{m}\right)$.

Our first assumption above is necessary to remove the global spatial configuration of features from the posterior calculation, which is straightforward from the mismatch rejection methods proposed. Even though we know that our second assumption is unrealistic, it is necessary, since the estimation of the joint probability $P\left(\mathcal{E} \mid T, \mathcal{O}_{m}\right)$ would require an extremely large number of training cases.

\subsection{Probabilistic Correspondences Based on Feature Similarity}

Using the image deformations in Appendix B and the database of random features in Appendix A, which can be found at http://computer.org/tpami/archives.htm it is possible to determine three properties of each model feature $\mathbf{f}_{l} \in \mathcal{O}_{m}$ (refer to [11] for more details): 1) the probability distribution of feature similarities, given a correct correspondence $\left.P_{\text {on }}\left(s_{f}(.) ; \mathbf{f}_{l}\right), 2\right)$ the probability distribution of feature similarities, given a false correspondence $P_{\text {off }}\left(s_{f}(.) ; \mathbf{f}_{l}\right)$, and 3) the probability of feature detection $P_{\text {det }}\left(\mathbf{f}_{l}\right)$. Using these properties, we compute the probabilistic correspondence, as explained later in Section 6.3.

\subsection{Probabilistic Correspondences Based on Semilocal Geometry}

The likelihood terms $P\left(e_{i} \mid T, \mathcal{O}_{m}\right)$ and $P\left(e_{i} \mid T, \neg \mathcal{O}_{m}\right)$ of each correspondence $e_{i}$ in $\mathcal{E}$ also involve feature value and semilocal geometric similarity. Since we assume that the pairwise relations are affected by a zero-mean Gaussian noise (see Section 5), only the variance of each pairwise relation in the model needs to be learned. Ideally, these variances should be estimated from real images of the same object, but that would require strong supervision in order to determine the locations of each model feature in each training image. Instead, we resorted to a simpler training procedure, where we use a single training image and artificially deform it (see deformations in Appendix B) so that the exact position of each model feature can be computed precisely. Let $\mathcal{O}_{m}$ represent the model features from model image $I_{m}$ and $\tilde{\mathcal{O}}_{m, d}$ be the features detected from the deformed version of image $I_{m}$, namely, $\tilde{I}_{m, d}$, using a deformation $d \in \mathcal{D} \mathcal{F}$. The correspondence set between these two sets is given by

$$
\begin{aligned}
\mathcal{N}_{m, d} & =\left\{\left(\mathbf{f}_{l}, \tilde{\mathbf{f}}_{l}\right) \mid \tilde{\mathbf{f}}_{l} \in \mathcal{O}_{m, d},\left\|\tilde{\mathbf{x}}_{l}-M(d) \mathbf{x}_{l}-\mathbf{b}(d)\right\|\right. \\
& \left.<\epsilon, \mathbf{f}_{l} \in \mathcal{K}\left(\tilde{\mathbf{f}}_{l}, \mathcal{O}_{m}, \kappa_{\mathcal{N}}\right)\right\},
\end{aligned}
$$

where $\mathcal{K}$, defined in (1), is the top- $\kappa_{\mathcal{N}}$ correspondences (here, $\kappa_{\mathcal{N}}=1$ ), $\epsilon$ was fixed at $\frac{\lambda_{d}}{4}=2.0$ pixels (as measured in the image $\left.\tilde{I}_{m, d}\right), \mathbf{x}_{l}$ is the position of feature $\mathbf{f}_{l}, \tilde{\mathbf{x}}_{l}$ is the position of feature $\tilde{\mathbf{f}}_{\mathrm{l}}$, and the transformation parameters $M(d)$ and $\mathbf{b}(d)$ are obtained from the deformation $d \in \mathcal{D F}$. 


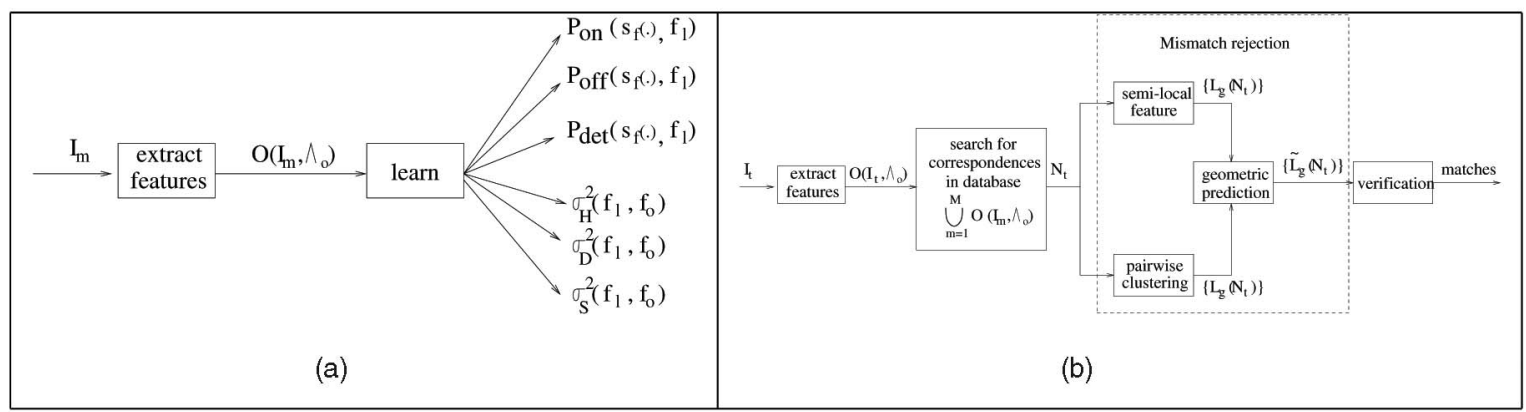

Fig. 14. Block diagrams of the learning and recognition procedures. (a) Block diagram of learning. (b) Block diagram of recognition.

Assuming that the uncertainties of the pairwise relations are normally distributed, we have

$$
\begin{aligned}
\sigma_{\mathcal{S}}^{2}\left(\mathbf{f}_{l}, \mathbf{f}_{o}\right) & =\operatorname{var}\left(\left\{\Delta \mathcal{S}_{l o}\left(\mathcal{N}_{m, d}\right)\right\}_{d \in \mathcal{D F}}\right), \\
\sigma_{\mathcal{D}}^{2}\left(\mathbf{f}_{l}, \mathbf{f}_{o}\right) & =\operatorname{var}\left(\left\{\Delta \mathcal{D}_{l o}\left(\mathcal{N}_{m, d}\right)\right\}_{d \in \mathcal{D F}}\right), \text { and } \\
\sigma_{\mathcal{H}}^{2}\left(\mathbf{f}_{l}, \mathbf{f}_{o}\right) & =\operatorname{var}\left(\left\{\Delta \mathcal{H}_{l o}\left(\mathcal{N}_{m, d}\right)\right\}_{d \in \mathcal{D F}}\right)
\end{aligned}
$$

for all deformations $d \in \mathcal{D} \mathcal{F}$, where var is the sample variance of the values in the set, and the pairwise relations between an object and its deformed version are provided by $\Delta \mathcal{S}_{l o}\left(\mathcal{N}_{m, d}\right), \Delta \mathcal{D}_{l o}\left(\mathcal{N}_{m, d}\right)$, and $\Delta \mathcal{H}_{l o}\left(\mathcal{N}_{m, d}\right)$ (see (3)). Therefore, for the term $P\left(e_{i} \mid T, \mathcal{O}_{m}\right)$, the idea is to use the geometric predictions defined in Section 5 to determine the likelihood of the correspondence $e_{i}$, and for $P\left(e_{i} \mid T, \neg \mathcal{O}_{m}\right)$, we simply assume a uniform distribution of the geometric configuration error.

\subsection{Final Verification}

Given a model $\mathcal{O}_{m}$, learned using the algorithm described above (see the block diagram in Fig. 14a), the model presence in a test image $I_{t}$ is determined as follows (see the block diagram in Fig. 14b). First, build the set of local features $\mathcal{O}_{t}$ from $I_{t}$, then search for similar local features in the database of models, thus forming the $\mathcal{N}_{m t}(1)$. Note that each test image feature is matched to $\kappa_{\mathcal{N}}$ model features and that it is possible that a model feature is matched to more than one test feature. We handle this kind of multiple correspondences originating from one feature in the model image by representing them as separate entities in the correspondence set. Given these correspondences, the mismatch rejection step forms a set of $G$ clusters $\left\{\tilde{\mathcal{L}}_{g}\left(\mathcal{N}_{t}\right)\right\}_{g=1}^{G}$ (see Sections 4.1 and 4.2.1). Each cluster is a hypothesis that a particular object is present in the image, so our goal is to determine if any of the clusters $\tilde{\mathcal{L}_{g}}\left(\mathcal{N}_{t}\right)$ actually represents an instance of the object $\mathcal{O}_{m}$. Let us first define the set of pairings for all model features $\mathbf{f}_{l} \in \mathcal{O}_{m}$ from group $\tilde{\mathcal{L}_{g}}\left(\mathcal{N}_{t}\right)$, as

$$
\begin{aligned}
& \mathcal{E}_{g}= \\
& \tilde{\mathcal{L}_{g}}\left(\mathcal{N}_{t}\right) \bigcup\left\{\left(\mathbf{f}_{l}, \emptyset\right) \mid \mathbf{f}_{l} \in \mathcal{O}_{m}, \neg \exists \tilde{\mathbf{f}}_{l} \in \mathcal{O}_{t} \text { s.t. }\left(\mathbf{f}_{l}, \tilde{\mathbf{f}}_{\mathrm{l}}\right) \in \tilde{\mathcal{L}_{g}}\left(\mathcal{N}_{t}\right)\right\} .
\end{aligned}
$$

Therefore, we compute the posterior (15) as follows:

1. $P\left(\mathcal{O}_{m}\right)$ is the prior expectation of the model presence and $P\left(\neg \mathcal{O}_{m}\right)=1-P\left(\mathcal{O}_{m}\right)$ (here, we assume that $\left.P\left(\mathcal{O}_{m}\right)=0.001\right)$.

2. $P\left(\mathcal{E}_{g} \mid T, \mathcal{O}_{m}\right) \approx \prod_{\left(\mathbf{f}_{l}, \tilde{\mathbf{f}}_{1}\right) \in \mathcal{E}_{g}} P\left(\left(\mathbf{f}_{l}, \tilde{\mathbf{f}}_{1}\right) \mid T, \mathcal{O}_{m}\right)$, where we have two cases: a. $\quad P\left(\left(\mathbf{f}_{l}, \emptyset\right) \in \mathcal{E}_{g} \mid T, \mathcal{O}_{m}\right) \approx\left(1-P_{\operatorname{det}}\left(\mathbf{x}_{l}\right)\right)+P_{\operatorname{det}}\left(\mathbf{x}_{l}\right) P_{\text {on }}$ $\left(s_{f} \tau_{s} ; \mathbf{f}_{l}\right)$, where $\tau_{s}$ is the threshold in (1), and $P_{\text {det }}\left(\mathbf{f}_{l}\right)$ and $P_{\text {on }}($.$) are defined in Section 6.1.$ The intuition is that if the model feature is not matched to a test feature, then either it was not detected (first term of the sum) or it was detected but not included in the correspondence set (second term).

b. $P\left(\left(\mathbf{f}_{l}, \tilde{\mathbf{f}}_{l}\right) \in \mathcal{E}_{g} \mid T, \mathcal{O}_{m}\right) \approx P_{\operatorname{det}}\left(\mathbf{f}_{l}\right) P_{\text {on }}\left(s_{f}\left(\mathbf{f}_{l}, \tilde{\mathbf{f}}_{l}\right) ; \mathbf{f}_{l}\right)$ $p\left(\mathbf{f}_{l}, \mathbf{f}_{1}\right)$, where $p($.$) is defined in (14). Here, we$ consider that for $\left(\mathbf{f}_{l}, \tilde{\mathbf{f}}_{1}\right) \in \mathcal{E}_{g}$, the feature has to be detected in the test image (first term of the multiplication), with a certain similarity value (second term) and geometric configuration (third term).

3. $P\left(\mathcal{E}_{g} \mid T, \neg \mathcal{O}_{m}\right)=\prod_{\left(\mathbf{f}_{l}, \tilde{\mathbf{f}}_{\mathrm{l}}\right) \in \mathcal{E}_{g}} P\left(\left(\mathbf{f}_{l}, \tilde{\mathbf{f}}_{\mathbf{l}}\right) \mid T, \neg \mathcal{O}_{m}\right)$, where we have two cases:

a. $\quad P\left(\left(\mathbf{f}_{l}, \emptyset\right) \in \mathcal{E}_{g} \mid T, \neg \mathcal{O}_{m}\right) \approx(1-0.032)+0.032\left(P_{\text {off }}\right.$ $\left.\left(s_{f}<\tau_{s} ; \mathbf{f}_{l}\right)\right)$, where the number 0.032 represents the average number of interest points per test image divided by the size of the image (see [8]), and $P_{\text {off }}($.$) is defined in Section 6.1. Similar to the$ case above, the likelihood of having an unmatched model feature, assuming that the model is not present, is approximated by the probability of general detection failure (first term) plus the likelihood of detection times the likelihood of not including the match in $\mathcal{E}_{g}$.

b. $\quad P\left(\left(\mathbf{f}_{l}, \tilde{\mathbf{f}}_{l}\right) \in \mathcal{E}_{g} \mid T, \neg \mathcal{O}_{m}\right) \approx(0.032) P_{\text {off }}\left(s_{f}\left(\tilde{\mathbf{f}}_{l}, \mathbf{f}_{l}\right) ; \mathbf{f}_{l}\right)$ $\frac{1}{\operatorname{size}(\mathcal{I})} \frac{1}{8} \frac{1}{2 \pi}$. In the last term, we assume a uniform distribution of position, main orientation, and scale, given a background feature. The intuition is that the likelihood of matching a model to a test feature in this case is related to the general feature detection, a high similarity to FP matches, and an arbitrary geometric configuration.

Finally, we accept a hypothesis if $P\left(\mathcal{O}_{m} \mid \mathcal{E}_{g}, T\right)$ is above a probability value, the number of correctly predicted matches (using (14)) is above a threshold, and the maximum distance between the test image features is bigger than a threshold; that is, assuming that $\tilde{\mathbf{x}}_{1}$ and $\tilde{\mathbf{x}}_{\mathrm{O}}$ are the positions of test image features $\tilde{\mathbf{f}}_{\mathrm{l}}$ and $\tilde{\mathbf{f}}_{\mathrm{o}}$, respectively, with $\left(\mathbf{f}_{l}, \tilde{\mathbf{f}}_{l}\right),\left(\mathbf{f}_{o}, \tilde{\mathbf{f}}_{\mathrm{o}}\right) \in \mathcal{E}_{g}$, we require

$$
\max _{\forall l, o}\left(\frac{\left\|\mathbf{x}_{l}-\mathbf{x}_{o}\right\|}{\sqrt{\sigma_{l}^{2}+\sigma_{o}^{2}}}\right)>\tau_{\mathcal{D}}
$$




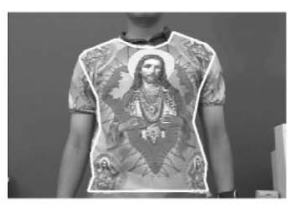

Torso

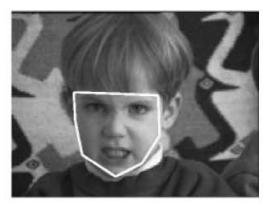

Kevin

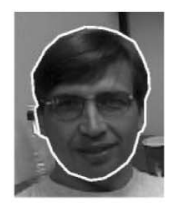

Dudek
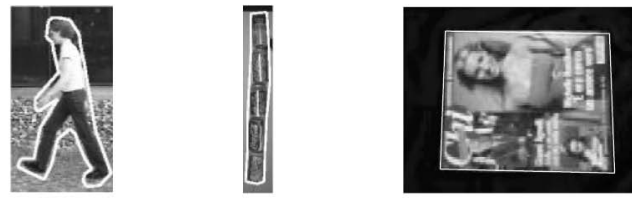

Hedvig Snake of Cans

Michelle [17]

Fig. 15. Models used for long-range motion. All the models are represented only by the features inside the contour around the object of interest.

(this is done to avoid a large number of features all in a small area of the image).

\section{EXPERIMENTS}

In this section, we show the qualitative and quantitative performance of our recognition algorithm by using the phasebased feature [8], both mismatch rejection methods, and the probabilistic verification. The following tasks are considered: 1) wide baseline stereo matching and 2) long-range motion matching. The main difference between the wide baseline stereo and the long-range motion experiments is that the former always involves the computation of the epipolar geometry, given a pair of images presenting a significant $3 \mathrm{D}$ rigid transformation, whereas the latter concerns matching pairs of images that might have suffered not only 3D rigid but also nonrigid deformations.

\subsection{Recognition Parameters}

Referring to the block diagram in Fig. 14b, the search for similar features (see (1)) in the model database involves two parameters: 1) the phase correlation threshold (here, $\left.\tau_{s}=0.75\right)$ and 2) the maximum number of nearest neighbors $\left(\kappa_{\mathcal{N}}=1\right)$. The following step is the mismatch rejection based on either the pairwise clustering or the semilocal feature. The parameters used for the mismatch rejection methods are the same as described in Section 4.3, where $\tau_{\mathrm{CCA}}=0.2$ for the pairwise grouping method (see (4)), and $\tau_{c}=0.5$ for the semilocal feature (see (6)). The acceptance of a hypothesis is evaluated in the verification step, which depends upon the posterior $P\left(\mathcal{O}_{m} \mid \mathcal{E}_{g}, T\right)>0.5$, the maximum distance between test image features being at least 20 percent of the maximum model diameter in pixels, and the number of correctly predicted matches being at least 3 percent of the total number of features of the model. The parameters above are found to provide a good balance between robustness to image deformations and to FPs, and they are kept fixed throughout the experiments.

\subsection{Long-Range Motion Results}

The long-range motion application is likely to be the most appropriate application for the system presented in this work. In fact, any task that involves the recognition and rough localization of textured objects that suffered severe 3D rigid and nonrigid deformations (including articulation) is well suited for this system. In this section, the model (see Fig. 15) is always represented by only one view of the object, and the system tries to find it throughout the sequence. We also provide a comparison using the Hough transform as a baseline method for eliminating mismatches in combination with the verification stage based on geometric prediction.

The sequences of the Torso, Hedvig, Kevin, and Dudek models (see samples in Fig. 16) are quite challenging due to the presence of nonrigid deformations, brightness, 3D rigid transformations, and partial occlusion. Fig. 16 shows the verification results using either the pairwise grouping or the semilocal feature methods to reject mismatches. Although we only show the most severely deformed samples in each sequence, it is interesting to see the quantitative performance of this system in each sequence, as shown in Table 1. We do not show the number of true negatives, since that number would be related to all possible data associations between the set of model and test features, which is equal to $\left|\mathcal{O}_{m}\right|^{\left|\mathcal{O}_{t}\right|}$, where both $\left|\mathcal{O}_{m}\right|$ and $\left|\mathcal{O}_{t}\right|$ are in the order of $10^{3}$. Also, in this table, we show the performance of the system using the Hough transform to reject mismatches followed by the geometric prediction in the verification stage.

The snake-of-cans model in Fig. 17 represents another challenging set of images that shows the articulated object in several different poses. Illumination changes are also present due to the highlights in the metal cans. Notice that both methods are quite robust in terms of articulate deformations. In contrast, the Hough transform provides poorer performance in these cases.

Finally, Fig. 18 shows the most challenging cases (in terms of nonrigid deformation) from the database of images designed by Ferrari et al. [17]. In general, the pairwise grouping and local features are more robust to nonrigid deformations than the Hough transform and consequently tend to include more correct matches in the final correspondence set. The runtime for the tasks of searching for correspondences, mismatch rejection, and verification varies between 5 and 10 seconds in nonoptimized Matlab code for all the cases presented in this section.

\subsection{Wide Baseline Stereo Results}

A wide baseline stereo problem involves two images, where a significant 3D rigid transformation took place between them, and the goal is to reliably compute their epipolar geometry. In order to robustly compute this epipolar geometry, we need a reasonably large number of matches situated on different planes of the scene. Using the same experimental setup introduced in Section 4.3.1, we focus on the computation of the $\mathbf{F}$ matrix and also on the number of trials $t$ necessary to make the probability of choosing at least one outlier in every trial of the RANSAC algorithm smaller 


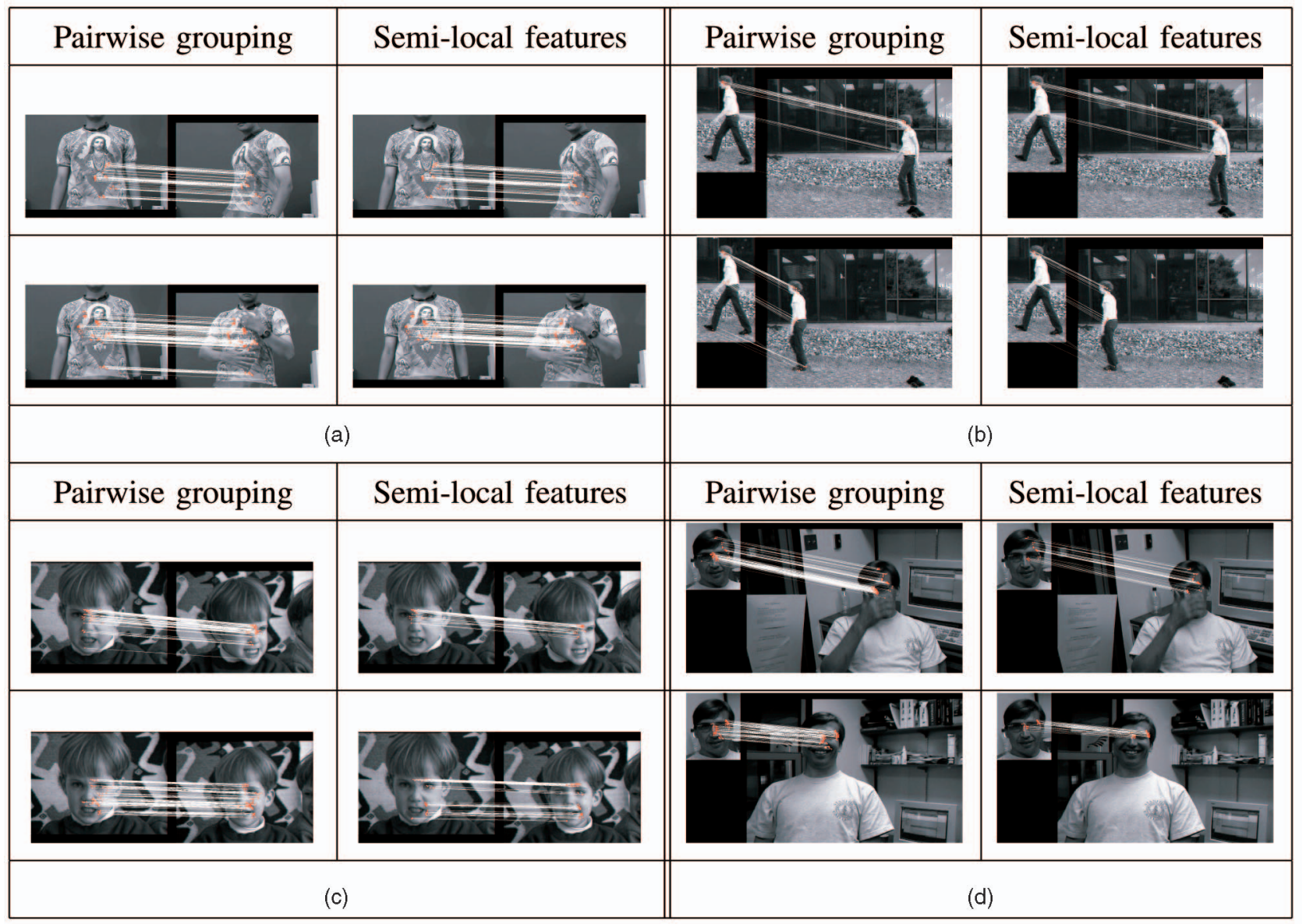

Fig. 16. Matchings for the Torso, Hedvig, Kevin, and Dudek models. The first and third columns show the verification results using pairwise grouping for rejecting mismatches and the second and fourth columns use the phase-based semilocal features. White lines are the correspondences between model and test images after verification. (a) Torso sequence. (b) Hedvig sequence. (c) Kevin sequence. (d) Dubek sequence.

TABLE 1

Performance of the Recognition Algorithm in Each Sequence

\begin{tabular}{|ccccccccccc|}
\hline Sequence & Length & \multicolumn{3}{c}{ True positives } & \multicolumn{3}{c}{ False positives } & \multicolumn{3}{c|}{ False negatives } \\
& & Pairwise & Semi-local & Hough & Pairwise & Semi-local & Hough & Pairwise & Semi-local & Hough \\
Dudek & 140 & 138 & 130 & 105 & 0 & 0 & 9 & 2 & 10 & 35 \\
Kevin & 120 & 120 & 109 & 111 & 0 & 0 & 12 & 0 & 11 & 9 \\
Hedvig & 33 & 30 & 31 & 28 & 0 & 0 & 1 & 3 & 2 & 5 \\
Torso & 148 & 148 & 147 & 148 & 0 & 0 & 8 & 0 & 1 & 0 \\
\hline
\end{tabular}

than 5 percent. We assume that the percentage of inliers is $p_{\text {crtmtc }}=\frac{i n}{i n+o u t}$, where $i n$ is the number of inliers in the set and out the number of outliers, and that the matrix $\mathbf{F}$ has 7 degrees of freedom. Using eight point correspondences to estimate $\mathrm{F}$, the probability of finding at least one mismatch in a randomly selected subset of eight correspondences from the initial set is $p_{\text {error }}=1-p_{\text {crtmtc }}^{8}$. As a result, the number of trials $t$ to make the probability of choosing at least one outlier in every trial of the RANSAC algorithm smaller than 5 percent is defined as $p_{\text {error }}^{t} \leq 0.05$, so $t$ can be determined by $t \leq\left\lceil\frac{\log _{2}(0.05)}{\log _{2}\left(p_{\text {error }}\right)}\right\rceil$.

Figs. 19 and 20 show the wide baseline stereo pairs for the Merton and Wadham sequences. Notice that both outlier rejection methods return a correspondence set with a high percentage of inliers, which is between 93 percent and 99 percent. This large proportion of true correspondences is likely to reduce the complexity of the algorithm to compute the $\mathbf{F}$ matrix. The average runtime for the tasks of searching for correspondences, mismatch rejection, and verification is around 10 seconds in nonoptimized Matlab code.

\section{Conclusions}

The use of spatial configuration of local features aims at reducing the number of mismatches in the correspondence set. This is desirable in order to decrease the complexity of the verification stage and to reduce the likelihood of FPs and false 


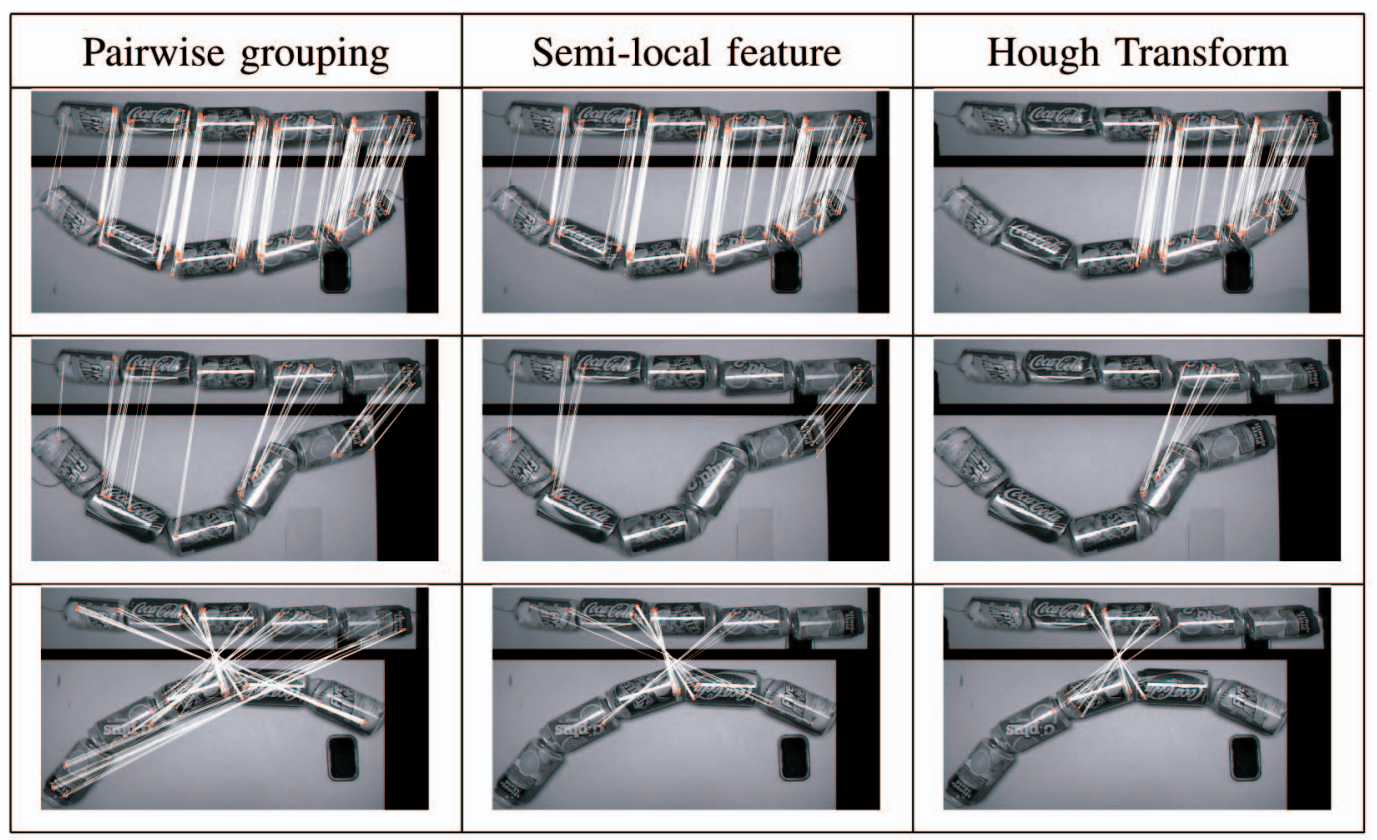

Fig. 17. Matchings for the snake-of-cans model.

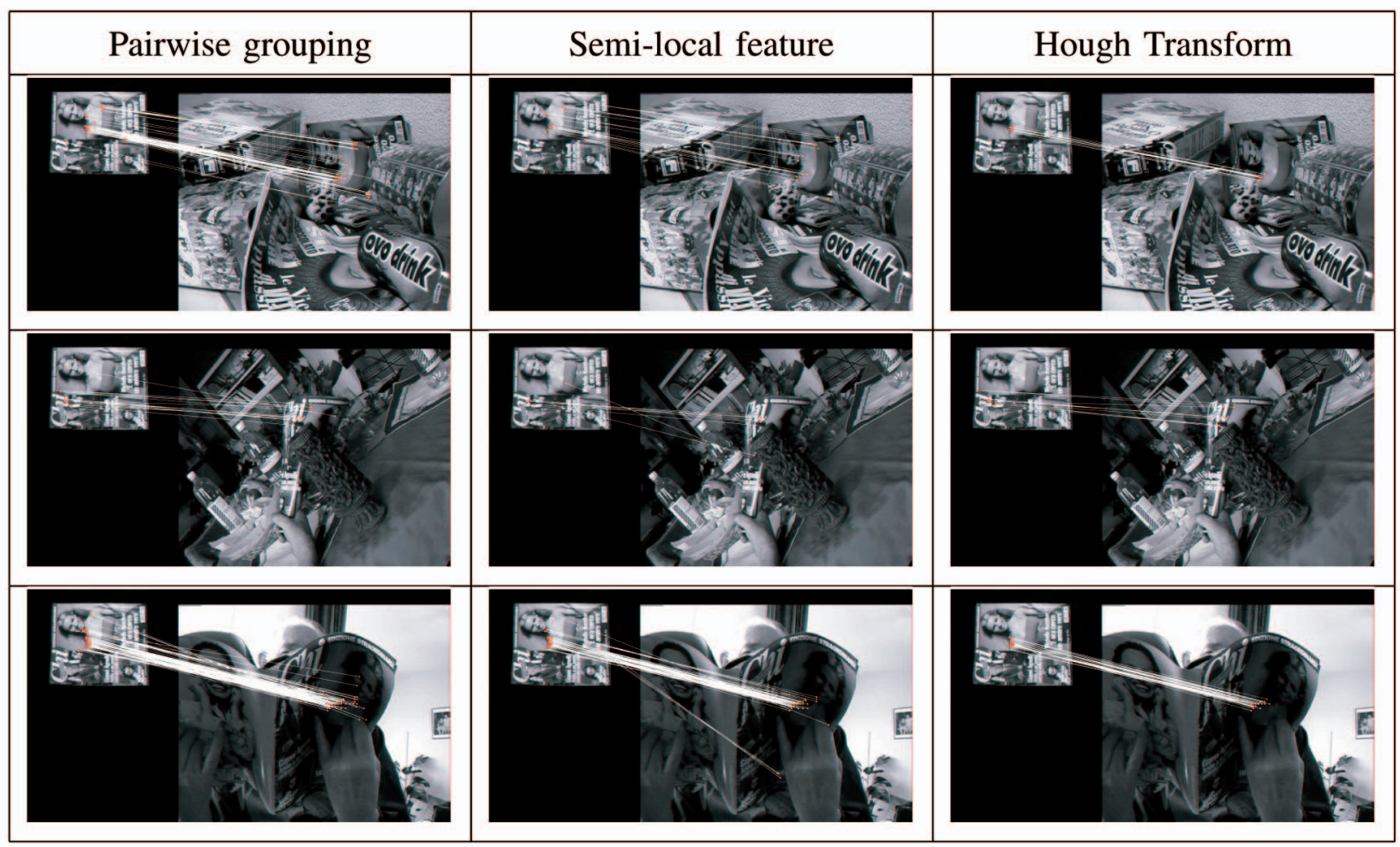

Fig. 18. Matchings for the Michelle model [17].

negatives. We proposed two methods to reject mismatches based on semilocal spatial information and another method to reject mismatches and to verify hypotheses based on the prediction of the geometric information of local features. We presented comparisons between our methods and the Hough clustering, which is a common mismatch rejection method based on the global spatial configuration of features, and the results show that our approaches are more robust to rigid transformations and nonrigid deformations. Also, our mismatch rejection methods are shown to have a time complexity roughly similar to that of the Hough transform. We also propose a new probabilistic verification that takes into account the semilocal spatial configuration of each feature and the feature similarity. Results on long-range matching and wide baseline stereo matching show the efficacy of the proposed method. 


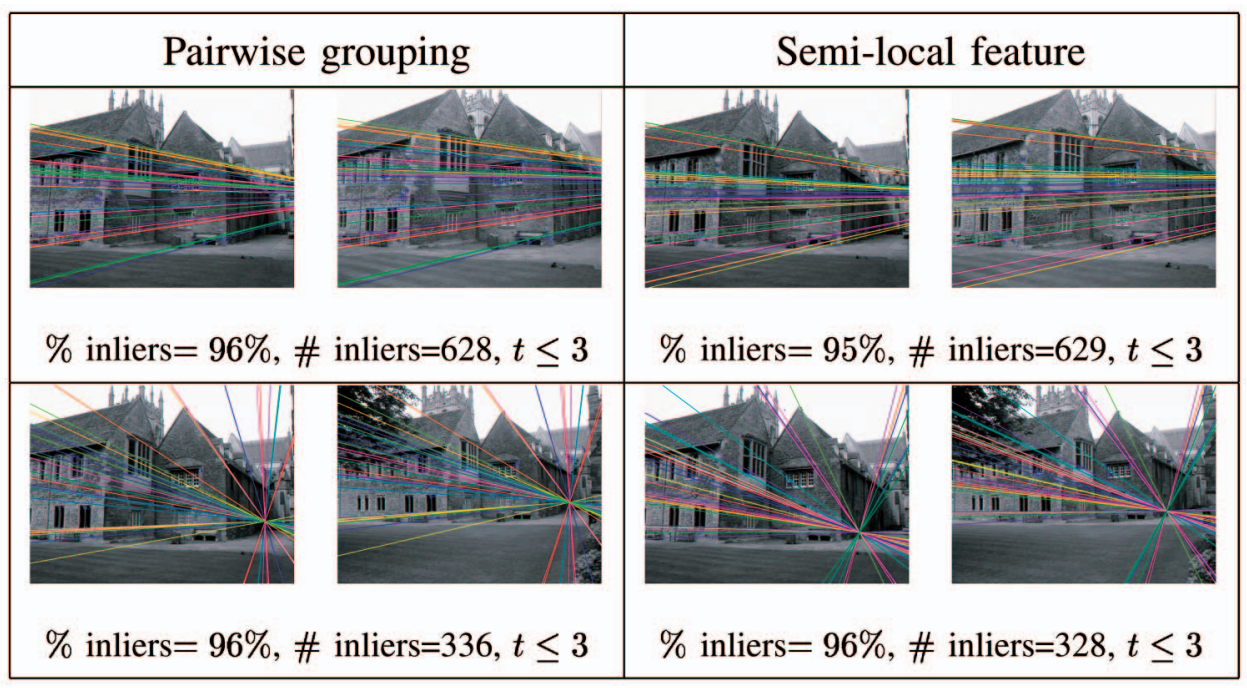

Fig. 19. Epipolar geometry for the Merton sequence. In the caption, we show the proportion of correct matches, given the $\mathbf{F}$ matrix computed ("percent correct matches"). Also, "\# correct matches" shows the total number of correct matches used, and " $\mathrm{t}$ " is the number of trials necessary to make the probability $p<0.05$ of choosing at least one mismatch in every trial of the RANSAC algorithm.

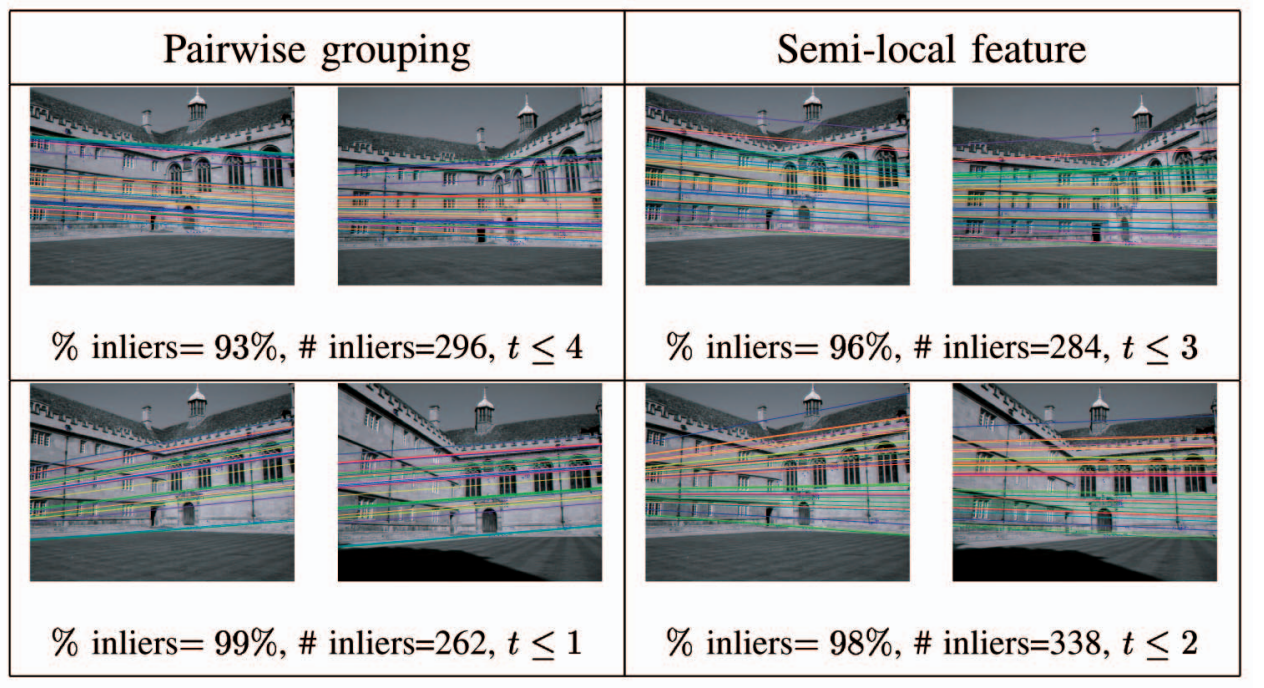

Fig. 20. Epipolar geometry for the Wadham sequence. See Fig. 19 for details on the captions.

\section{REFERENCES}

[1] S. Agarwal and D. Roth, "Learning a Sparse Representation for Object Detection," Proc. Seventh European Conf. Computer Vision, pp. 113-130, 2002.

[2] Y. Amit and D. Geman, "A Computational Model for Visual Selection," Neural Computation, vol. 11, pp. 1691-1715, 1999.

[3] H. Barrow and R. Popplestone, "Relational Descriptions in Picture Processing," Machine Intelligence, vol. 6, 1971.

[4] S. Belongie, J. Malik, and J. Puzicha, "Shape Matching and Object Recognition Using Shape Contexts," IEEE Trans. Pattern Analysis and Machine Intelligence, vol. 24, no. 4, pp. 509-522, Apr. 2002.

[5] A. Berg, T. Berg, and J. Malik, "Shape Matching and Object Recognition Using Low Distortion Correspondences," Proc. IEEE Conf. Computer Vision and Pattern Recognition, 2005.

[6] K.L. Boyer and S. Sarkar, Perceptual Organization for Artificial Vision Systems. Kluwer Academic, 2000.

[7] R. Brooks, "Model-Based 3D Interpretations of 2D Images," IEEE Trans. Pattern Analysis and Machine Intelligence, vol. 5, no. 2, pp. 140150, 1983.

[8] G. Carneiro and A. Jepson, "Multi-Scale Phase-Based Local Features," Proc. IEEE Conf. Computer Vision and Pattern Recognition, June 2003.
[9] G. Carneiro and A. Jepson, "Flexible Spatial Models for Grouping Local Image Features," Proc. IEEE Conf. Computer Vision and Pattern Recognition, June 2004.

[10] G. Carneiro and A. Jepson, "Pruning Local Feature Correspondences Using Shape Context," Proc. 17th IEEE Int'l Conf. Pattern Recognition, Aug. 2004.

[11] G. Carneiro and A. Jepson, "The Distinctiveness, Detectability, and Robustness of Local Image Features," Proc. IEEE Conf. Computer Vision and Pattern Recognition, June 2005.

[12] C. Huang, O. Camps, and T. Kanungo, "Object Recognition Using Appearance-Based Parts and Relations," Proc. IEEE Conf. Computer Vision and Pattern Recognition, pp. 877-883, 1997.

[13] O. Chum, J. Matas, and S. Obdrzalek, "Epipolar Geometry from Three Correspondences," Proc. Eighth Computer Vision Winter Workshop, 2003.

[14] S. Dickinson, A. Pentland, and A. Rosenfeld, "3D Shape Recovery Using Distributed Aspect Matching," IEEE Trans. Pattern Analysis and Machine Intelligence, vol. 14, no. 2, pp. 174-198, Feb. 1992.

[15] P. Felzenszwalb and D. Huttenlocher, "Efficient Matching of Pictorial Structures," Proc. IEEE Conf. Computer Vision and Pattern Recognition, pp. 66-75, 2000.

[16] R. Fergus, P. Perona, and A. Zisserman, "Object Class Recognition by Unsupervised Scale-Invariant Learning," Proc. IEEE Conf. Computer Vision and Pattern Recognition, 2003. 
[17] V. Ferrari, T. Tuytelaars, and L.V. Gool, "Simultaneous Object Recognition and Segmentation by Image Exploration," Proc. Eighth European Conf. Computer Vision, 2004.

[18] D. Fleet, Measurement of Image Velocity. Kluwer Academic, 1992.

[19] W. Freeman and E. Adelson, "The Design and Use of Steerable Filters," IEEE Trans. Pattern Analysis and Machine Intelligence, vol. 13, no. 9, pp. 891-906, Sept. 1991.

[20] C. Harris and M. Stephens, "A Combined Corner and Edge Detector," Proc. Fourth Alvey Vision Conf., 1988.

[21] R. Hartley and A. Zisserman, Multiple View Geometry in Computer Vision. Cambridge Univ. Press, 2000.

[22] B. Huet and E. Hancock, "Relational Object Recognition from Large Structural Libraries," Pattern Recognition, vol. 35, pp. 18951915, 2002

[23] M. Lades, J.C. Vorbruggen, J. Buhmann, J. Lange, C.v.d. Malsburg, R.P. Wurtz, and W. Konen, "Distortion Invariant Object Recognition in the Dynamic Link Architecture," IEEE Trans. Computers, vol. 42, pp. 300-311, 1993.

[24] D. Lowe, "Three-Dimensional Object Recognition from Single Two-Dimensional Images," Artificial Intelligence, vol. 31, no. 3, pp. 355-395, 1987.

[25] D. Lowe, "Object Recognition from Local Scale-Invariant Features," Proc. Seventh Int'l Conf. Computer Vision, pp. 1150-1157, Sept. 1999.

[26] D. Lowe, "Local Feature View Clustering for 3D Object Recognition," Proc. IEEE Conf. Computer Vision and Pattern Recognition, 2001.

[27] K. Mikolajczyk, A. Zisserman, and C. Schmid, "Shape Recognition with Edge-Based Features," Proc. 14th British Machine Vision Conf., 2003.

[28] E. Mortensen, H. Deng, and L. Shapiro, "A Sift Descriptor with Global Context," Proc. IEEE Conf. Computer Vision and Pattern Recognition (CVPR '05), 2005.

[29] H. Murase and S. Nayar, "Visual Learning and Recognition of 3D Objects from Appearance," Int'l J. Computer Vision, vol. 14, no. 1, pp. 5-24, 1995.

[30] J. Pilet, V. Lepetit, and P. Fua, "Real-Time Non-Rigid Surface Detection," Proc. IEEE Conf. Computer Vision and Pattern Recognition, 2005.

[31] A. Pope and D. Lowe, "Probabilistic Models of Appearance for 3D Object Recognition," Int'l J. Computer Vision, vol. 40, no. 2, pp. 149167, 2000.

[32] P. Pritchett and A. Zisserman, "Wide Baseline Stereo Matching," Proc. Sixth Int'l Conf. Computer Vision, pp. 754-760, 1998.

[33] F. Schaffalitzky and A. Zisserman, "Automated Scene Matching in Movies," Proc. Int'l Conf. Image and Video Retrieval, pp. 186-197, 2002.

[34] C. Schmid, "A Structured Probabilistic Model for Recognition," Proc. IEEE Conf. Computer Vision and Pattern Recognition, pp. 485490, 1999.

[35] C. Schmid and R. Mohr, "Local Grayvalue Invariants for Image Retrieval," IEEE Trans. Pattern Analysis and Machine Intelligence, vol. 19, no. 5, pp. 530-535, May 1997.

[36] A. Shokoufandeh, S. Dickinson, C. Jonsson, L. Bretzner, and T. Lindeberg, "On the Representation and Matching of Qualitative Shape at Multiple Scales," Proc. Seventh European Conf. Computer Vision, 2002.

[37] A. Shokoufandeh, I. Marsic, and S. Dickinson, "View-Based Object Recognition Using Saliency Maps," Image and Vision Computing, vol. 17, pp. 445-460, 1999.

[38] J. Sivic, F. Schaffalitzky, and A. Zisserman, “Object Level Grouping for Video Shots," Proc. Eighth European Conf. Computer Vision, 2004.

[39] D. Tell and S. Carlsson, "Wide Baseline Point Matching Using Affine Invariants Computed from Intensity Profiles," Proc. Sixth European Conf. Computer Vision, pp. 814-828, 2000.

[40] D. Tell and S. Carlsson, "Combining Appearance and Topology for Wide Baseline Matching," Proc. Seventh European Conf. Computer Vision, pp. 68-81, 2002.

[41] P. Torr and D. Murray, "The Development and Comparison of Robust Methods for Estimating the Fundamental Matrix," Int'l J. Computer Vision, vol. 24, no. 3, pp. 271-300, 1997.

[42] T. Tuytelaars and L. Van Gool, "Wide Baseline Stereo Matching Based on Local, Affinely Invariant Regions," Proc. 11th British Machine Vision Conf., 2000.
[43] M. Weber, M. Welling, and P. Perona, "Unsupervised Learning of Models for Recognition," Proc. Sixth European Conf. Computer Vision, pp. 18-32, 2000.

[44] S. Yu, R. Gross, and J. Shi, "Concurrent Object Recognition and Segmentation by Graph Partitioning," Neural Information Processing Systems, Dec. 2002.

[45] Z. Zhang, R. Deriche, O.D. Faugeras, and Q. Luong, "A Robust Technique for Matching Two Uncalibrated Images through the Recovery of the Unknown Epipolar Geometry," Artificial Intelligence, vol. 78, nos. 1-2, pp. 87-119, 1995.

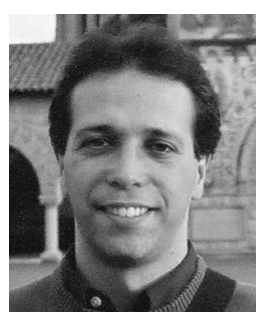

Gustavo Carneiro received the $\mathrm{PhD}$ degree in computer science from the University of Toronto. $\mathrm{He}$ is a research scientist in the Department of Integrated Data Systems, Siemens Corporate Research. He joined Siemens in January of 2006. In 2005, he received a postdoctoral fellowship from the National Sciences and Engineering Research Council of Canada (NSERC) to work at the Laboratory of Computational Intelligence, University of British Columbia. In 2004, he was a postdoctoral fellow at the Statistical Visual Computing Laboratory, University of California, San Diego, where the work presented in this paper was developed. His main research interests include visual pattern recognition in computer vision, medical image analysis, and image processing.

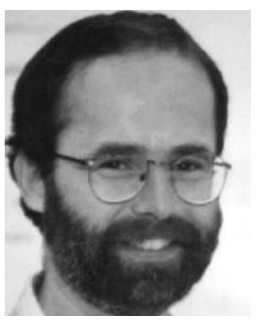

Allan D. Jepson received the BSc degree in mathematics from the University of British Columbia in 1976 and the PhD degree in applied mathematics from the California Institute of Technology in 1980. He spent two years as a postdoctoral fellow at the Department of Mathematics, Stanford University, and then joined the faculty of the Department of Computer Science, University of Toronto, in 1982. From 1989 to 1995, he was a scholar of the Canadian Institute of Advanced Research. His current research interests include image motion estimation, image understanding, and perceptual inference. He is a member of the IEEE Computer Society.

$\triangleright$ For more information on this or any other computing topic, please visit our Digital Library at www.computer.org/publications/dlib. 